\title{
THE GOULD’s BELT VERY LARGE ARRAY SURVEY. I. THE OPHIUCHUS COMPLEX
}

\author{
Sergio A. DziB ${ }^{1}$, Laurent Loinard ${ }^{1,2}$, Amy J. Mioduszewsit ${ }^{3}$, Luis F. Rodríguez ${ }^{1,4}$, Gisela N. Ortiz-León ${ }^{1}$, \\ Gerardo Pech ${ }^{1}$, Juana L. Rivera ${ }^{1}$, Rosa M. Torres ${ }^{5}$, Andrew F. Boden ${ }^{6}$, Lee Hartmann $^{7}$, \\ Neal J. Evans II ${ }^{8}$, Cesar Briceño ${ }^{9}$, AND John Tobin ${ }^{10,11}$ \\ ${ }^{1}$ Centro de Radioastronomía y Astrofísica, Universidad Nacional Autónoma de México Apartado Postal 3-72, \\ 58090 Morelia, Michoacán, Mexico; s.dzib@crya.unam.mx \\ 2 Max Planck Institut für Radioastronomie, Auf dem Hügel 69, D-53121 Bonn, Germany \\ ${ }^{3}$ National Radio Astronomy Observatory, Domenici Science Operations Center, 1003 Lopezville Road, Socorro, NM 87801, USA \\ ${ }^{4}$ King Abdulaziz University, P.O. Box 80203, Jeddah 21589, Saudi Arabia \\ ${ }^{5}$ Paul Harris 9065, Las Condes, Santiago, Chile \\ ${ }^{6}$ Division of Physics, Math, and Astronomy, California Institute of Technology, 1200 East California Boulevard, Pasadena, CA 91125, USA \\ ${ }^{7}$ Department of Astronomy, University of Michigan, 500 Church Street, Ann Arbor, MI 48105, USA \\ ${ }^{8}$ Department of Astronomy, The University of Texas at Austin, 1 University Station, C1400, Austin, TX 78712, USA \\ ${ }^{9}$ Centro de Investigaciones de Astronomía, Mérida 5101-A, Venezuela \\ ${ }^{10}$ National Radio Astronomy Observatory, Charlottesville, VA 22903, USA \\ Received 2013 June 5; accepted 2013 July 18; published 2013 September 5
}

\begin{abstract}
We present large-scale $\left(\sim 2000 \operatorname{arcmin}^{2}\right)$, deep $(\sim 20 \mu \mathrm{Jy})$, high-resolution $\left(\sim 1^{\prime \prime}\right)$ radio observations of the Ophiuchus star-forming complex obtained with the Karl G. Jansky Very Large Array at $\lambda=4$ and $6 \mathrm{~cm}$. In total, 189 sources were detected, 56 of them associated with known young stellar sources, and 4 with known extragalactic objects; the other 129 remain unclassified, but most of them are most probably background quasars. The vast majority of the young stars detected at radio wavelengths have spectral types $\mathrm{K}$ or $\mathrm{M}$, although we also detect four objects of $\mathrm{A} / \mathrm{F} / \mathrm{B}$ types and two brown dwarf candidates. At least half of these young stars are non-thermal (gyrosynchrotron) sources, with active coronas characterized by high levels of variability, negative spectral indices, and (in some cases) significant circular polarization. As expected, there is a clear tendency for the fraction of non-thermal sources to increase from the younger (Class 0/I or flat spectrum) to the more evolved (Class III or weak line T Tauri) stars. The young stars detected both in X-rays and at radio wavelengths broadly follow a Güdel-Benz relation, but with a different normalization than the most radioactive types of stars. Finally, we detect a $\sim 70 \mathrm{mJy}$ compact extragalactic source near the center of the Ophiuchus core, which should be used as gain calibrator for any future radio observations of this region.
\end{abstract}

Key words: astrometry - magnetic fields - radiation mechanisms: non-thermal - radio continuum: stars techniques: interferometric

Online-only material: color figures

\section{INTRODUCTION}

Gould's Belt (see Poppel 1997 for a recent review) is a flattened local Galactic structure, about $1 \mathrm{kpc}$ in size, that contains much of the recent star-forming activity in the solar neighborhood. In particular, it comprises the nearest sites of active star formation, i.e., the Taurus, Ophiuchus, and Orion molecular complexes. This paper is the first in a series that will describe the results of deep, large-scale radio observations of these nearby regions of star formation; it will focus on the Ophiuchus complex.

A combination of several properties conspires to make Ophiuchus one of the most interesting targets for star formation studies (see Wilking et al. 2008 for a recent review). Its proximity (120 pc; Loinard et al. 2008) ensures high linear spatial resolution, and enables the detection of fainter objects (e.g., brown dwarfs) than in other regions. In addition, the stellar population associated with the central dark cloud Lynds 1688 (often called the Ophiuchus core; see Figure 1) provides us with an example of a young $(\tau<0.5 \mathrm{Myr}$; Wilking et al. 2008) stellar cluster that probes a mode of star formation intermediate between the more isolated situation exemplified by Taurus, and the more clustered mode typically found in higher-mass regions. As a consequence, Ophiuchus has been one of the best-studied

\footnotetext{
${ }^{11}$ Hubble Fellow
}

regions of star formation. In the last decade alone, very detailed surveys of its stellar population have been obtained in X-rays (Imanishi et al. 2001; Gagné et al. 2004; Ozawa et al. 2005; Sciortino et al. 2006), near-infrared (Alves de Oliveira et al. 2010; Barsony et al. 2012), and mid-infrared (Padgett et al. 2008). The properties of the circumstellar disks around these young stars have been studied in the mid-infrared (KesslerSilacci et al. 2006; Geers et al. 2006; Lahuis et al. 2007) and millimeter/submillimeter regimes (Andrews \& Williams 2007). Finally, the distribution of the interstellar material has also been studied in detail thanks to millimeter spectroscopy (Ridge et al. 2006), submillimeter continuum observations (Motte et al. 1998; Johnstone et al. 2000; Stanke et al. 2006; Young et al. 2006), and far-infrared imaging (Padgett et al. 2008).

Numerous radio observations have also been obtained, but the characterization of the Ophiuchus complex at radio wavelengths remains significantly less systematic than in other frequency bands. Following the early work by Brown \& Zuckerman (1975) and Falgarone \& Gilmore (1981), the first large-scale observations were obtained with the Very Large Array (VLA) in its $\mathrm{C}$ and $\mathrm{D}$ configurations at 1.4 and $5 \mathrm{GHz}$ by André et al. (1987) and Stine et al. (1988). While most of the detected radio sources were background extragalactic objects, roughly a dozen could be confidently identified with young stars in the Ophiuchus cluster. The next major step came with the deep $5 \mathrm{GHz}$ VLA observations of the Ophiuchus core, again in the 


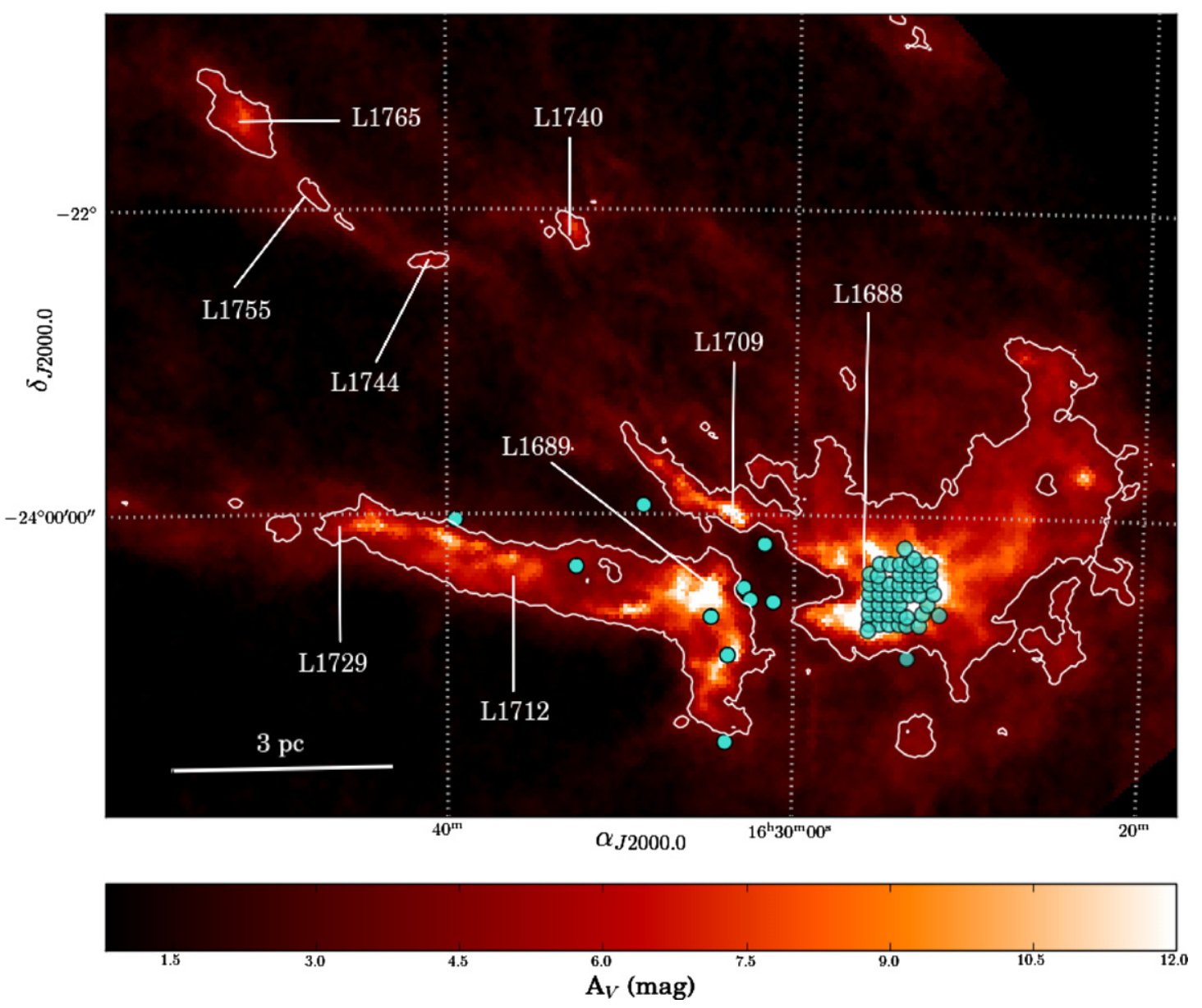

Figure 1. Extinction map of the Ophiuchus complex obtained as part of the COMPLETE project (Ridge et al. 2006) based on the 2MASS data (Skrutskie et al. 2006). The Lynds dark clouds in the regions are indicated, and a linear distance is provided (assuming a distance of 120 pc to the entire region—Loinard et al. 2008). The turquoise circles indicate the areas mapped with the VLA for the survey presented here. The diameter of each circle is $6^{\prime}$ and corresponds to the primary beam of the VLA at $7.5 \mathrm{GHz}$. Note that the field of view, and therefore also the total mapped area, at $4.5 \mathrm{GHz}$, is significantly larger.

(A color version of this figure is available in the online journal.)

C and D configurations, reported by Leous et al. (1991). These observations targeted two specific fields, but reached a noise level several times better than that of previous observations. A total of 35 compact radio sources were identified, most of them associated with young stellar objects (YSOs: Wilking et al. 2001). More recently, Gagné et al. (2004) reported the detection at $6 \mathrm{~cm}$ of a dozen additional compact sources, but it is not clear what fraction of these sources might be associated with young stars. Finally, a number of observations (with better sensitivity and/or higher angular resolution) have been obtained toward specific objects (particularly the prototypical Class 0/I sources IRAS 16293-2422, VLA 1623, and YLW15-Wootten 1989; Chandler et al. 2005; Loinard et al. 2007, 2013; Andre et al. 1993; Ward-Thompson et al. 2011; Girart et al. 2000, 2004).

The analysis of the radio properties of young stars in Ophiuchus has helped clarify the origin of the radio emission produced by YSOs in general. For sources with flat, Class I or Class II infrared spectral energy distributions, the radio emission (at $v \lesssim 30 \mathrm{GHz}$ ) tends to be of thermal bremsstrahlung (free-free) origin, ${ }^{12}$ and to trace the dense central base of ionized winds (e.g., Rodríguez 1999). This type of emission is characterized

\footnotetext{
12 The Class 0 source IRAS $16293-2422$ is somewhat of an oddity where thermal dust emission appears to remain the dominant emission process well into the centimeter regime (Loinard et al. 2013 and reference therein). This, however, is a very unusual situation, presumably partly related to the large amount of dense gas and dust around IRAS 16293-2422; we do not expect to find any source of this type in the present survey.
}

by a spectral index $\alpha$ (defined such that the flux depends on frequency as $\left.S_{v} \propto v^{\alpha}\right)$ between -0.1 and $+2(\alpha=+0.6$ for the classical case of a partially optically thick isotropic wind; Panagia 1973), and only presents moderate variability $(\lesssim 20 \%)$. In contrast, for Class III sources, the emission is generally nonthermal (gyrosynchrotron) and related to magnetic activity near the stellar surface. This kind of emission often (but not always) exhibits strong variability and some level of circular polarization (Dulk 1985). The spectral index for gyrosynchrotron emission depends on the energy distribution of the electrons, and can vary between -2 and +2 (Dulk 1985), although a slightly negative index occurs in many cases. Furthermore, non-thermal radiation mechanisms produce high brightness emission $\left(T_{b} \gtrsim 10^{6}\right.$ to $\left.10^{7} \mathrm{~K}\right)$ confined to a very compact region $\left(\lesssim 10 R_{\odot}\right)$. As a consequence, it is detectable even in very long baseline interferometry (VLBI) radio experiments, as shown by Lestrade et al. (1991), Phillips et al. (1991), or Andre et al. (1992). It should be mentioned that the separation between thermal (free-free) and non-thermal (gyrosynchrotron) emission at the Class II/III boundary is not a sharp one as a number of Class II and even a few Class I sources have been found to emit non-thermal radiation (e.g., Forbrich et al. 2007; Dzib et al. 2010; Deller et al. 2013).

In the present article, we report on new radio observations of the Ophiuchus complex which largely surpass previously published studies thanks to a combination of high sensitivity, 
subarcsecond angular resolution, and large field of view. These data will be used both to discuss the population of radio sources in Ophiuchus, and the relation between radio properties of YSOs and their characteristics at other wavelengths. The observations are described in Section 2; the results are presented in Section 3, and analyzed in Sections 4 and 5. Our conclusions are provided in Section 6.

\section{OBSERVATIONS}

The observations were obtained with the Karl G. Jansky VLA of the National Radio Astronomy Observatory in its CnB and $\mathrm{B}$ configurations. Two frequency sub-bands, each $1 \mathrm{GHz}$ wide, and centered at 4.5 and $7.5 \mathrm{GHz}$, respectively, were recorded simultaneously. The observations were obtained on three different epochs (2011 February 17/19, April 3/4, and May 4/6) typically separated from one another by a month. This dual frequency, multi-epoch strategy was chosen to enable the characterization of the spectral index and variability of the detected sources, and to help in the identification of the emission mechanisms (thermal versus non-thermal).

Since our aim was to examine the distribution and properties of radio sources in the Ophiuchus complex in a statistically meaningful fashion, it was important to systematically map a large area of the complex, including those regions known to harbor a high density of young stars. The region of highest stellar density is associated with the dark cloud Lynds 1688 (the Ophiuchus core). We mapped this area, which contains over 400 YSOs, using a mosaic of 47 VLA pointings (see Figure 1). Ten additional pointings were selected to cover regions associated with other dust clouds (particularly L1689, but also L1709, L1712, and L1729; see Figure 1) located to the east of the Ophiuchus core (these clouds are known collectively as the Ophiuchus streamers).

The FWHM of the primary beam (i.e., the field of view) of the VLA has a diameter of $10^{\prime}$ at $4.5 \mathrm{GHz}$, and $6^{\prime}$ at $7.5 \mathrm{GHz}$. As a consequence, the 10 individual fields targeting the streamers cover an area of $785 \operatorname{arcmin}^{2}$ at $4.5 \mathrm{GHz}$ and $283 \operatorname{arcmin}^{2}$ at $7.5 \mathrm{GHz}$. The spacing between the individual pointings of the mosaic observed toward L1688 follows a somewhat irregular pattern chosen to optimize the compromise between uniform sensitivity and inclusion of the largest possible number of known young stars. The total area covered by these fields is $1185 \operatorname{arcmin}^{2}$ at $4.5 \mathrm{GHz}$ and $878 \operatorname{arcmin}^{2}$ at $7.5 \mathrm{GHz}$.

Each observing session was organized as follows. The standard flux calibrator 3C 286 was first observed for $\sim 10$ minutes. We subsequently spent one minute on the phase calibrator J1626-2951 followed by a series of three target pointings, spending three minutes on each. This phase calibrator/target sequence was repeated until all target fields were observed. Thus, three minutes were spent on each target field for each epoch. The data were edited, calibrated, and imaged in a standard fashion using the Common Astronomy Software Applications package (CASA).

In the 10 individual fields associated with the streamers, the noise level reached for each epoch was 61 and $52 \mu \mathrm{Jy} \mathrm{beam}^{-1}$ at $4.5 \mathrm{GHz}$ and $7.5 \mathrm{GHz}$, respectively. For the mosaicked region, on the other hand, a nearly uniform noise level of $26 \mu \mathrm{Jy}$ beam $^{-1}$ is obtained at both frequencies. The frequency independence of the noise is the result of two effects that compensate each other: while the noise in individual fields is somewhat better at $7.5 \mathrm{GHz}$, the larger field of view at lower frequency results in a larger overlap between fields which reduces the noise in the final mosaic. To produce images with improved sensitivity, the three epochs were combined, resulting in noise levels of 30 and $25 \mu \mathrm{Jy}$ beam $^{-1}$ at $4.5 \mathrm{GHz}$ and $7.5 \mathrm{GHz}$, respectively, for the individual (streamers) fields, and $17 \mu \mathrm{Jy}_{\text {beam }}{ }^{-1}$ at both frequencies for the mosaic. The angular resolution of the observations is of the order of $1^{\prime \prime}$.

To test for circular polarization, we produced images of the $V$ Stokes parameter in the inner quarter (in area) of the primary beam at each frequency. At larger distances from the field center, polarization measurements are unreliable as beam squint (the separation of the $R$ and $L$ beams on the sky) can create artificial circular polarization signals.

\section{RESULTS}

The first step is, of course, to identify sources in our observations. This was done using the images corresponding to the concatenation of the three epochs, which provide the highest sensitivity. The criteria used to consider a detection as firm were: (1) sources with reported counterparts and a flux larger than four times the $\sigma$ noise of the area, or (2) sources with a flux larger than five times the $\sigma$ noise of the area and without reported counterparts. From this, a total of 189 sources were detected (see Table 1). To reflect the fact that these sources were found as part of the Gould's Belt Very Large Array Survey, a source with coordinates hhmmss.ss-ddmmss.s will be named GBS-VLA Jhhmmss.ss-ddmmss.s.

The flux of each source at 4.5 and $7.5 \mathrm{GHz}$ are given in Columns 2 and 4 of Table 1 ). Two sources of uncertainties on the fluxes are included: (1) the error that results from the statistical noise in the images, and (2) a systematic uncertainty of 5\% resulting from possible errors in the absolute flux calibration. An estimation of the radio spectral index of each source (given in Column 6 of Table 1) was obtained from the fluxes measured in each sub-band (at 4.5 and $7.5 \mathrm{GHz}$ ). To calculate the errors on the spectral indices, the two sources of errors (statistical and systematic) on the flux at each frequency were added in quadrature and the final error was obtained using standard error propagation theory.

Once the sources were identified in the concatenated images, we searched for them in the images obtained from the individual epochs. ${ }^{13}$ An estimate of the level of variability of the sources was measured by comparing the fluxes measured at the three epochs. Specifically, we calculated, for each source and at each frequency, the difference between the highest and lowest measured fluxes, and normalized by the maximum flux. The resulting values, expressed in percent, are given in Columns 3 and 5 of Table 1 . Circular polarization was confidently detected in seven of our targets (Table 2).

Having identified the radio sources in the region mapped, our next step was to try to determine which type of object they are associated with. In our specific case, the two overwhelmingly dominant possibilities are young stars and extragalactic sources. ${ }^{14}$ We searched the literature for previous radio detections, and for counterparts at X-ray, optical, near-, and midinfrared wavelengths. The search was done in SIMBAD, and accessed all the major catalogs (listed explicitly in the footnote of Table 3). Note that the Spitzer c2d catalog includes cross-references to other major catalogs which were taken into

\footnotetext{
13 We also searched the individual epochs for objects that might be present there although they are not detected in the averaged data, but found no such object.

14 Although we cannot fully rule out the possibility that other objects might contaminate the sample (a planetary nebula, for instance), the probability of such an occurrence is very low.
} 
Table 1

Radio Sources Detected in Ophiuchus

\begin{tabular}{|c|c|c|c|c|c|}
\hline \multirow[t]{2}{*}{ GB-VLA Name } & \multicolumn{4}{|c|}{ Flux Properties } & \multirow[t]{2}{*}{ Spectral Index } \\
\hline & $f_{4.5}(\mathrm{mJy})$ & Var. $(\%)$ & $f_{7.5}(\mathrm{mJy})$ & Var. $(\%)$ & \\
\hline J162540.94-244147.2 & $(4.39 \pm 0.43 \pm 0.22) \times 10^{-1}$ & $16.2 \pm 11.9$ & $(2.94 \pm 0.42 \pm 0.15) \times 10^{-1}$ & $>56.2 \pm 12.3$ & $-0.81 \pm 0.38$ \\
\hline J162542.48-242143.7 & $(3.79 \pm 0.32 \pm 0.19) \times 10^{-1}$ & $4.3 \pm 13.7$ & & & \\
\hline J162547.68-243735.7 & $(4.19 \pm 0.21 \pm 0.21) \times 10^{-1}$ & $28.5 \pm 8.6$ & $(4.06 \pm 0.28 \pm 0.20) \times 10^{-1}$ & $51.3 \pm 15.0$ & $-0.06 \pm 0.22$ \\
\hline $\mathrm{J} 162548.96-244049.5$ & $(6.49 \pm 0.34 \pm 0.32) \times 10^{-1}$ & $41.7 \pm 5.4$ & $(2.92 \pm 0.32 \pm 0.15) \times 10^{-1}$ & $>66.9 \pm 4.3$ & $-1.61 \pm 0.28$ \\
\hline J162550.51-243914.7 & $(3.57 \pm 0.25 \pm 0.18) \times 10^{-1}$ & $78.2 \pm 5.3$ & $(2.84 \pm 0.18 \pm 0.14) \times 10^{-1}$ & $41.4 \pm 13.1$ & $-0.46 \pm 0.24$ \\
\hline J162550.84-243719.6 & $(8.00 \pm 1.00 \pm 0.40) \times 10^{-2}$ & $>43.6 \pm 4.7$ & $<0.06$ & & $<-0.58 \pm 0.14$ \\
\hline J162551.91-242917.0 & $(1.71 \pm 0.35 \pm 0.09) \times 10^{-1}$ & $21.4 \pm 19.7$ & $(1.15 \pm 0.24 \pm 0.06) \times 10^{-1}$ & $>17.4 \pm 37.5$ & $-0.8 \pm 0.61$ \\
\hline $\mathrm{J} 162556.09-243015.3$ & $(6.72 \pm 0.15 \pm 0.34) \times 10^{-1}$ & $31.4 \pm 3.3$ & $(6.76 \pm 0.24 \pm 0.34) \times 10^{-1}$ & $10.5 \pm 4.5$ & $0.01 \pm 0.17$ \\
\hline $\mathrm{J} 162557.51-243032.1$ & $(4.61 \pm 0.17 \pm 0.23) \times 10^{-1}$ & $55.8 \pm 3.3$ & $(4.07 \pm 0.17 \pm 0.20) \times 10^{-1}$ & $56.1 \pm 4.5$ & $-0.25 \pm 0.18$ \\
\hline J162603.01-242336.4 & $(2.67 \pm 0.09 \pm 0.13) \times 10^{+0}$ & $81.7 \pm 0.6$ & $(2.52 \pm 0.09 \pm 0.13) \times 10^{+0}$ & $84.9 \pm 1.0$ & $-0.12 \pm 0.18$ \\
\hline J162605.29-243436.6 & $<0.05$ & & $(1.05 \pm 0.15 \pm 0.05) \times 10^{-1}$ & $33.9 \pm 27.2$ & $>1.34 \pm 0.14$ \\
\hline $\mathrm{J} 162607.63-242741.7$ & $(2.10 \pm 0.21 \pm 0.10) \times 10^{-1}$ & $>79.5 \pm 1.0$ & $(1.01 \pm 0.21 \pm 0.05) \times 10^{-1}$ & $>67.0 \pm 3.6$ & $-1.48 \pm 0.49$ \\
\hline $\mathrm{J} 162607.63-243648.9$ & $(5.83 \pm 0.17 \pm 0.29) \times 10^{-1}$ & $37.1 \pm 3.1$ & $(3.67 \pm 0.14 \pm 0.18) \times 10^{-1}$ & $39.4 \pm 7.4$ & $-0.94 \pm 0.17$ \\
\hline $\mathrm{J} 162608.04-242523.1$ & $(1.03 \pm 0.14 \pm 0.05) \times 10^{-1}$ & $37.9 \pm 15.6$ & $<0.05$ & & $<-1.3 \pm 0.14$ \\
\hline J162610.32-242054.9 & $(1.00 \pm 0.12 \pm 0.05) \times 10^{-1}$ & $>56.1 \pm 5.6$ & $(1.60 \pm 0.22 \pm 0.08) \times 10^{-1}$ & $53.4 \pm 16.7$ & $0.95 \pm 0.40$ \\
\hline $\mathrm{J} 162610.55-242853.3$ & $(2.09 \pm 0.15 \pm 0.10) \times 10^{-1}$ & $24.2 \pm 10.0$ & $(1.68 \pm 0.19 \pm 0.08) \times 10^{-1}$ & $43.0 \pm 17.0$ & $-0.44 \pm 0.31$ \\
\hline J162611.08-242907.4 & $(4.51 \pm 0.10 \pm 0.23) \times 10^{-1}$ & $16.2 \pm 4.4$ & $(2.98 \pm 0.16 \pm 0.15) \times 10^{-1}$ & $42.4 \pm 6.5$ & $-0.84 \pm 0.18$ \\
\hline J162611.79-243716.4 & $(1.66 \pm 0.12 \pm 0.08) \times 10^{-1}$ & $20.9 \pm 15.5$ & $(1.11 \pm 0.12 \pm 0.06) \times 10^{-1}$ & $30.9 \pm 17.5$ & $-0.81 \pm 0.30$ \\
\hline $\mathrm{J} 162615.67-243421.2$ & $(9.17 \pm 0.07 \pm 0.46) \times 10^{+0}$ & $\ldots$ & $(6.23 \pm 0.12 \pm 0.31) \times 10^{+0}$ & & Extended \\
\hline $\mathrm{J} 162616.84-242223.5$ & $(3.37 \pm 0.15 \pm 0.17) \times 10^{-1}$ & $>75.9 \pm 0.8$ & $(3.60 \pm 0.24 \pm 0.18) \times 10^{-1}$ & $>83.1 \pm 0.3$ & $0.13 \pm 0.22$ \\
\hline $\mathrm{J} 162617.66-244014.5$ & $(4.65 \pm 0.26 \pm 0.23) \times 10^{-1}$ & $46.3 \pm 5.0$ & $(3.31 \pm 0.25 \pm 0.17) \times 10^{-1}$ & $30.9 \pm 11.5$ & $-0.69 \pm 0.24$ \\
\hline J162620.56-243523.4 & $(7.30 \pm 1.50 \pm 0.36) \times 10^{-2}$ & $>40.2 \pm 23.0$ & $<0.05$ & $\ldots$ & $<-0.61 \pm 0.14$ \\
\hline $\mathrm{J} 162621.72-242250.7$ & $(2.38 \pm 0.17 \pm 0.12) \times 10^{-1}$ & $28.8 \pm 11.2$ & $(3.04 \pm 0.29 \pm 0.15) \times 10^{-1}$ & $>13.2 \pm 14.2$ & $0.49 \pm 0.28$ \\
\hline $\mathrm{J} 162622.38-242253.3$ & $(2.02 \pm 0.06 \pm 0.10) \times 10^{+0}$ & $68.6 \pm 1.6$ & $(1.42 \pm 0.07 \pm 0.07) \times 10^{+0}$ & $85.1 \pm 1.4$ & $-0.71 \pm 0.18$ \\
\hline J162623.40-243223.6 & $(1.25 \pm 0.15 \pm 0.06) \times 10^{-1}$ & $>37.9 \pm 11.4$ & $<0.06$ & $\ldots$ & $<-1.48 \pm 0.14$ \\
\hline J162623.57-242439.6 & $(1.24 \pm 0.17 \pm 0.06) \times 10^{-1}$ & $33.5 \pm 16.2$ & $(1.15 \pm 0.08 \pm 0.06) \times 10^{-1}$ & $>38.2 \pm 6.0$ & $-0.15 \pm 0.34$ \\
\hline J162624.08-241613.5 & $(1.92 \pm 0.15 \pm 0.10) \times 10^{-1}$ & $22.3 \pm 9.6$ & $(3.44 \pm 0.28 \pm 0.17) \times 10^{-1}$ & $46.6 \pm 6.5$ & $1.18 \pm 0.27$ \\
\hline J162625.62-242429.2 & $(2.18 \pm 0.14 \pm 0.11) \times 10^{-1}$ & $17.6 \pm 15.2$ & $(1.98 \pm 0.23 \pm 0.10) \times 10^{-1}$ & $>47.2 \pm 6.3$ & $-0.19 \pm 0.30$ \\
\hline J162626.03-244923.7 & $(1.73 \pm 0.05 \pm 0.09) \times 10^{+1}$ & & & & Extended \\
\hline $\mathrm{J} 162626.31-242430.2$ & $(1.89 \pm 0.34 \pm 0.09) \times 10^{-1}$ & $63.1 \pm 11.0$ & $(1.89 \pm 0.34 \pm 0.09) \times 10^{-1}$ & $44.7 \pm 14.6$ & $-0.0 \pm 0.53$ \\
\hline J162626.39-242430.9 & $(8.70 \pm 3.00 \pm 0.43) \times 10^{-2}$ & $\ldots{ }^{a}$ & $(1.25 \pm 0.25 \pm 0.06) \times 10^{-1}$ & $\ldots{ }^{\mathrm{a}}$ & $0.73 \pm 0.82$ \\
\hline $\mathrm{J} 162629.63-242317.2$ & $(2.28 \pm 0.14 \pm 0.11) \times 10^{-1}$ & $43.3 \pm 10.3$ & $(1.24 \pm 0.18 \pm 0.06) \times 10^{-1}$ & $>64.2 \pm 9.4$ & $-1.23 \pm 0.35$ \\
\hline $\mathrm{J} 162629.67-241905.8$ & $(2.67 \pm 0.17 \pm 0.13) \times 10^{-1}$ & $57.4 \pm 6.0$ & $(2.27 \pm 0.21 \pm 0.11) \times 10^{-1}$ & $69.9 \pm 5.2$ & $-0.33 \pm 0.27$ \\
\hline J162630.15-243132.4 & $(1.08 \pm 0.20 \pm 0.05) \times 10^{-1}$ & $>59.2 \pm 8.6$ & $<0.05$ & $\ldots$ & $<-1.4 \pm 0.14$ \\
\hline $\mathrm{J} 162630.59-242023.0$ & $(9.80 \pm 1.30 \pm 0.49) \times 10^{-2}$ & $36.9 \pm 17.1$ & $(6.40 \pm 1.70 \pm 0.32) \times 10^{-2}$ & $\ldots^{a}$ & $-0.86 \pm 0.62$ \\
\hline J162631.28-241832.9 & $(2.42 \pm 0.26 \pm 0.12) \times 10^{-1}$ & $43.8 \pm 10.6$ & $(1.39 \pm 0.15 \pm 0.07) \times 10^{-1}$ & $8.8 \pm 30.6$ & $-1.12 \pm 0.34$ \\
\hline J162631.34-243341.8 & $(2.22 \pm 0.56 \pm 0.11) \times 10^{-1}$ & $37.1 \pm 19.4$ & $(1.66 \pm 0.26 \pm 0.08) \times 10^{-1}$ & $>67.9 \pm 11.9$ & $-0.59 \pm 0.62$ \\
\hline J162632.78-241627.5 & $(9.30 \pm 1.50 \pm 0.46) \times 10^{-2}$ & $38.8 \pm 18.7$ & $(1.42 \pm 0.24 \pm 0.07) \times 10^{-1}$ & $32.1 \pm 18.4$ & $0.86 \pm 0.49$ \\
\hline J162633.16-245246.7 & $(8.03 \pm 0.27 \pm 0.40) \times 10^{-1}$ & $30.7 \pm 5.5$ & $(9.18 \pm 0.43 \pm 0.46) \times 10^{-1}$ & $49.3 \pm 5.3$ & $0.27 \pm 0.18$ \\
\hline J162633.48-241215.9 & $(2.12 \pm 0.03 \pm 0.11) \times 10^{+0}$ & $17.4 \pm 1.5$ & $(9.27 \pm 0.32 \pm 0.46) \times 10^{-1}$ & $38.1 \pm 5.3$ & $-1.67 \pm 0.16$ \\
\hline J162634.17-242328.4 & $(7.98 \pm 0.25 \pm 0.40) \times 10^{+0}$ & $12.0 \pm 3.4$ & $(7.07 \pm 0.34 \pm 0.35) \times 10^{+0}$ & $23.6 \pm 5.3$ & $-0.24 \pm 0.18$ \\
\hline J162634.47-241656.8 & $(9.20 \pm 0.70 \pm 0.46) \times 10^{-2}$ & $>41.0 \pm 3.0$ & $<0.04$ & $\ldots$ & $<-1.44 \pm 0.14$ \\
\hline $\mathrm{J} 162634.95-242655.3$ & $(1.97 \pm 0.19 \pm 0.10) \times 10^{-1}$ & $30.1 \pm 12.9$ & $(1.00 \pm 0.13 \pm 0.05) \times 10^{-1}$ & $40.5 \pm 13.9$ & $-1.37 \pm 0.36$ \\
\hline $\mathrm{J} 162635.33-242405.2$ & $(6.50 \pm 0.38 \pm 0.33) \times 10^{-1}$ & $9.3 \pm 9.3$ & $(3.29 \pm 0.33 \pm 0.16) \times 10^{-1}$ & $20.3 \pm 18.6$ & $-1.38 \pm 0.27$ \\
\hline J162636.96-243755.9 & $(4.00 \pm 0.26 \pm 0.20) \times 10^{-1}$ & $12.8 \pm 10.7$ & $(1.75 \pm 0.24 \pm 0.09) \times 10^{-1}$ & $25.4 \pm 15.5$ & $-1.67 \pm 0.34$ \\
\hline J162637.27-244553.9 & $(2.91 \pm 0.33 \pm 0.15) \times 10^{-1}$ & $52.8 \pm 8.4$ & $(1.81 \pm 0.26 \pm 0.09) \times 10^{-1}$ & $>78.0 \pm 3.0$ & $-0.96 \pm 0.40$ \\
\hline J162638.99-244529.0 & $(3.78 \pm 0.19 \pm 0.19) \times 10^{-1}$ & $12.8 \pm 10.5$ & $(2.29 \pm 0.50 \pm 0.11) \times 10^{-1}$ & $>24.6 \pm 26.4$ & $-1.01 \pm 0.47$ \\
\hline J162639.00-243052.8 & $(4.05 \pm 0.27 \pm 0.20) \times 10^{-1}$ & $43.6 \pm 9.1$ & $(2.37 \pm 0.19 \pm 0.12) \times 10^{-1}$ & $21.4 \pm 14.8$ & $-1.08 \pm 0.25$ \\
\hline $\mathrm{J} 162639.70-241609.7$ & $(1.19 \pm 0.16 \pm 0.06) \times 10^{-1}$ & $15.3 \pm 31.0$ & $(6.60 \pm 1.40 \pm 0.33) \times 10^{-2}$ & $\ldots^{\mathrm{a}}$ & $-1.19 \pm 0.53$ \\
\hline J162641.11-245855.8 & $(6.45 \pm 0.26 \pm 0.32) \times 10^{-1}$ & $67.8 \pm 3.3$ & $(8.25 \pm 0.27 \pm 0.41) \times 10^{-1}$ & $81.0 \pm 2.3$ & $0.5 \pm 0.18$ \\
\hline J162642.44-242626.1 & $(2.66 \pm 0.06 \pm 0.13) \times 10^{+0}$ & $88.0 \pm 0.5$ & $(2.48 \pm 0.10 \pm 0.12) \times 10^{+0}$ & $92.3 \pm 1.2$ & $-0.14 \pm 0.17$ \\
\hline $\mathrm{J} 162642.53-244628.3$ & $(9.57 \pm 0.17 \pm 0.48) \times 10^{-1}$ & $51.3 \pm 3.2$ & $(8.31 \pm 0.43 \pm 0.42) \times 10^{-1}$ & $35.4 \pm 4.5$ & $-0.29 \pm 0.18$ \\
\hline J162643.76-241633.4 & $(1.31 \pm 0.04 \pm 0.07) \times 10^{+0}$ & $45.6 \pm 1.8$ & $(1.35 \pm 0.04 \pm 0.07) \times 10^{+0}$ & $46.0 \pm 1.8$ & $0.07 \pm 0.17$ \\
\hline J162646.36-242002.0 & $(5.53 \pm 0.21 \pm 0.28) \times 10^{-1}$ & $15.3 \pm 6.4$ & $(7.68 \pm 0.20 \pm 0.38) \times 10^{-1}$ & $21.6 \pm 5.7$ & $0.66 \pm 0.17$ \\
\hline $\mathrm{J} 162647.17-245157.4$ & $(5.91 \pm 0.25 \pm 0.30) \times 10^{-1}$ & $67.5 \pm 5.3$ & $(3.51 \pm 0.84 \pm 0.18) \times 10^{-1}$ & $>39.9 \pm 14.0$ & $-1.05 \pm 0.51$ \\
\hline $\mathrm{J} 162647.23-243620.3$ & $(4.18 \pm 0.15 \pm 0.21) \times 10^{-1}$ & $21.5 \pm 6.9$ & $(4.87 \pm 0.24 \pm 0.24) \times 10^{-1}$ & $22.0 \pm 7.9$ & $0.31 \pm 0.19$ \\
\hline J162647.32-245852.6 & $(1.50 \pm 0.25 \pm 0.07) \times 10^{-1}$ & $>12.2 \pm 16.1$ & $<0.13$ & $\ldots$ & $<-0.29 \pm 0.14$ \\
\hline J162647.57-245754.8 & $(5.75 \pm 0.62 \pm 0.29) \times 10^{-1}$ & $16.6 \pm 13.3$ & $(3.08 \pm 0.28 \pm 0.15) \times 10^{-1}$ & $37.4 \pm 9.8$ & $-1.26 \pm 0.32$ \\
\hline J162649.23-242003.3 & $(9.95 \pm 0.27 \pm 0.50) \times 10^{-1}$ & $82.0 \pm 2.0$ & $(7.44 \pm 0.40 \pm 0.37) \times 10^{-1}$ & $85.0 \pm 2.3$ & $-0.59 \pm 0.19$ \\
\hline J162649.90-245617.5 & $(2.02 \pm 0.15 \pm 0.10) \times 10^{-1}$ & $35.7 \pm 11.6$ & $(1.87 \pm 0.18 \pm 0.09) \times 10^{-1}$ & $33.3 \pm 21.8$ & $-0.16 \pm 0.28$ \\
\hline $\mathrm{J} 162651.69-241441.5$ & $(2.10 \pm 0.16 \pm 0.10) \times 10^{-1}$ & $>78.9 \pm 0.9$ & $(1.47 \pm 0.17 \pm 0.07) \times 10^{-1}$ & $>71.2 \pm 2.3$ & $-0.72 \pm 0.31$ \\
\hline J162653.38-241105.8 & $(2.83 \pm 0.20 \pm 0.14) \times 10^{-1}$ & $55.0 \pm 6.8$ & $(2.76 \pm 0.16 \pm 0.14) \times 10^{-1}$ & $33.1 \pm 7.6$ & $-0.05 \pm 0.23$ \\
\hline J162657.84-244201.6 & $(1.74 \pm 0.18 \pm 0.09) \times 10^{-1}$ & $35.9 \pm 11.7$ & $(1.61 \pm 0.16 \pm 0.08) \times 10^{-1}$ & $50.6 \pm 11.6$ & $-0.16 \pm 0.32$ \\
\hline J162658.25-243738.5 & $(1.12 \pm 0.11 \pm 0.06) \times 10^{-1}$ & $57.3 \pm 11.1$ & $(5.40 \pm 1.30 \pm 0.27) \times 10^{-2}$ & $\ldots{ }^{a}$ & $-1.47 \pm 0.54$ \\
\hline J162658.38-242130.5 & $(2.04 \pm 0.17 \pm 0.10) \times 10^{-1}$ & $>79.4 \pm 0.8$ & $(1.62 \pm 0.28 \pm 0.08) \times 10^{-1}$ & $>85.8 \pm 0.7$ & $-0.47 \pm 0.41$ \\
\hline
\end{tabular}


Table 1

(Continued)

\begin{tabular}{|c|c|c|c|c|c|}
\hline \multirow[t]{2}{*}{ GB-VLA Name } & \multicolumn{4}{|c|}{ Flux Properties } & \multirow[t]{2}{*}{ Spectral Index } \\
\hline & $f_{4.5}(\mathrm{mJy})$ & Var. $(\%)$ & $f_{7.5}(\mathrm{mJy})$ & Var. $(\%)$ & \\
\hline J162659.16-243458.9 & $(5.51 \pm 0.16 \pm 0.28) \times 10^{-1}$ & $10.3 \pm 6.4$ & $(7.02 \pm 0.24 \pm 0.35) \times 10^{-1}$ & $12.5 \pm 4.9$ & $0.49 \pm 0.17$ \\
\hline $\mathrm{J} 162659.96-243639.5$ & $(1.42 \pm 0.20 \pm 0.07) \times 10^{-1}$ & $34.5 \pm 9.0$ & $<0.05$ & $\ldots$ & $<-1.95 \pm 0.14$ \\
\hline J162659.99-241910.0 & $<0.10$ & $\ldots$ & $(5.35 \pm 0.59 \pm 0.27) \times 10^{-1}$ & $>84.5 \pm 1.0$ & $>3.39 \pm 0.14$ \\
\hline $\mathrm{J} 162700.00-242640.3$ & $(7.11 \pm 0.15 \pm 0.36) \times 10^{+1}$ & $12.6 \pm 1.8$ & $(7.09 \pm 0.38 \pm 0.35) \times 10^{+1}$ & $17.3 \pm 2.7$ & $-0.01 \pm 0.18$ \\
\hline J162700.02-243537.6 & $(2.45 \pm 0.13 \pm 0.12) \times 10^{-1}$ & $24.0 \pm 12.4$ & $(1.91 \pm 0.13 \pm 0.10) \times 10^{-1}$ & $46.5 \pm 11.9$ & $-0.5 \pm 0.23$ \\
\hline $\mathrm{J} 162702.11-243842.5$ & $(2.20 \pm 0.17 \pm 0.11) \times 10^{-1}$ & $>55.5 \pm 4.3$ & $(2.24 \pm 0.16 \pm 0.11) \times 10^{-1}$ & $68.6 \pm 7.3$ & $0.04 \pm 0.26$ \\
\hline J162702.15-241927.8 & $(5.48 \pm 0.19 \pm 0.27) \times 10^{-1}$ & $6.4 \pm 8.7$ & $(3.09 \pm 0.16 \pm 0.15) \times 10^{-1}$ & $19.3 \pm 11.8$ & $-1.16 \pm 0.19$ \\
\hline J162702.33-243727.3 & $(1.02 \pm 0.16 \pm 0.05) \times 10^{-1}$ & $>43.0 \pm 9.7$ & $(1.21 \pm 0.14 \pm 0.06) \times 10^{-1}$ & $>28.4 \pm 10.2$ & $0.35 \pm 0.42$ \\
\hline $\mathrm{J} 162702.36-242724.8$ & $(3.62 \pm 0.19 \pm 0.18) \times 10^{-1}$ & $>4.5 \pm 10.5$ & $(2.32 \pm 0.25 \pm 0.12) \times 10^{-1}$ & $>39.7 \pm 5.3$ & $-0.9 \pm 0.28$ \\
\hline $\mathrm{J} 162705.16-242007.8$ & $(2.54 \pm 0.22 \pm 0.13) \times 10^{-1}$ & $27.9 \pm 13.7$ & $(1.41 \pm 0.15 \pm 0.07) \times 10^{-1}$ & $>43.6 \pm 6.7$ & $-1.19 \pm 0.31$ \\
\hline $\mathrm{J} 162705.25-243629.8$ & $(1.46 \pm 0.30 \pm 0.07) \times 10^{-1}$ & $5.6 \pm 30.7$ & $(1.33 \pm 0.14 \pm 0.07) \times 10^{-1}$ & $29.6 \pm 15.1$ & $-0.19 \pm 0.49$ \\
\hline J162705.96-242618.9 & $(3.85 \pm 0.30 \pm 0.19) \times 10^{-1}$ & $>76.8 \pm 1.5$ & $(2.89 \pm 0.21 \pm 0.14) \times 10^{-1}$ & $>6.4 \pm 7.2$ & $-0.58 \pm 0.26$ \\
\hline J162709.41-243719.0 & $(2.51 \pm 0.15 \pm 0.13) \times 10^{-1}$ & $30.9 \pm 12.1$ & $(3.57 \pm 0.16 \pm 0.18) \times 10^{-1}$ & $24.2 \pm 8.9$ & $0.71 \pm 0.21$ \\
\hline $\mathrm{J} 162711.29-243722.5$ & $(1.45 \pm 0.12 \pm 0.07) \times 10^{-1}$ & $20.6 \pm 13.9$ & $(8.00 \pm 1.00 \pm 0.40) \times 10^{-2}$ & $>44.8 \pm 13.8$ & $-1.2 \pm 0.33$ \\
\hline J162713.06-241817.0 & $(3.00 \pm 0.18 \pm 0.15) \times 10^{-1}$ & $41.2 \pm 6.0$ & $(1.79 \pm 0.18 \pm 0.09) \times 10^{-1}$ & $21.3 \pm 14.4$ & $-1.04 \pm 0.28$ \\
\hline J162713.62-242226.1 & $(1.31 \pm 0.20 \pm 0.07) \times 10^{-1}$ & $42.7 \pm 24.8$ & $(1.08 \pm 0.26 \pm 0.05) \times 10^{-1}$ & $>31.8 \pm 24.8$ & $-0.39 \pm 0.59$ \\
\hline J162714.71-243919.7 & $(1.71 \pm 0.18 \pm 0.09) \times 10^{-1}$ & $>43.6 \pm 4.7$ & $<0.07$ & $\ldots$ & $<-1.8 \pm 0.14$ \\
\hline J162715.69-243845.7 & $(2.20 \pm 0.16 \pm 0.11) \times 10^{-1}$ & $24.4 \pm 13.2$ & $(2.36 \pm 0.28 \pm 0.12) \times 10^{-1}$ & $35.1 \pm 10.7$ & $0.14 \pm 0.32$ \\
\hline $\mathrm{J} 162717.38-243616.7$ & $(1.20 \pm 0.14 \pm 0.06) \times 10^{-1}$ & $22.5 \pm 18.5$ & $<0.05$ & & $<-1.61 \pm 0.14$ \\
\hline $\mathrm{J} 162718.17-242852.9$ & $(3.78 \pm 0.07 \pm 0.19) \times 10^{+0}$ & $68.7 \pm 1.0$ & $(3.14 \pm 0.09 \pm 0.16) \times 10^{+0}$ & $65.2 \pm 1.6$ & $-0.38 \pm 0.16$ \\
\hline J162718.25-243334.8 & $(2.99 \pm 0.28 \pm 0.15) \times 10^{-1}$ & $>45.8 \pm 3.0$ & $(1.14 \pm 0.14 \pm 0.06) \times 10^{-1}$ & $>53.1 \pm 17.7$ & $-1.95 \pm 0.34$ \\
\hline $\mathrm{J} 162719.34-243130.4$ & $(1.21 \pm 0.18 \pm 0.06) \times 10^{-1}$ & $>28.6 \pm 21.1$ & $(8.50 \pm 1.20 \pm 0.43) \times 10^{-2}$ & $\ldots{ }^{a}$ & $-0.71 \pm 0.44$ \\
\hline $\mathrm{J} 162719.50-244140.3$ & $(1.60 \pm 0.37 \pm 0.08) \times 10^{-1}$ & $>42.4 \pm 12.0$ & $(8.70 \pm 1.20 \pm 0.43) \times 10^{-2}$ & $>56.6 \pm 5.5$ & $-1.23 \pm 0.56$ \\
\hline J162721.81-244335.9 & $(3.66 \pm 0.23 \pm 0.18) \times 10^{-1}$ & $76.1 \pm 3.3$ & $(3.42 \pm 0.22 \pm 0.17) \times 10^{-1}$ & $>81.8 \pm 0.9$ & $-0.14 \pm 0.23$ \\
\hline $\mathrm{J} 162721.97-242940.0$ & $(1.47 \pm 0.24 \pm 0.07) \times 10^{-1}$ & $45.9 \pm 17.9$ & $(1.32 \pm 0.16 \pm 0.07) \times 10^{-1}$ & $41.1 \pm 13.0$ & $-0.22 \pm 0.43$ \\
\hline $\mathrm{J} 162722.96-242236.6$ & $(2.09 \pm 0.15 \pm 0.10) \times 10^{-1}$ & $66.8 \pm 10.6$ & $<0.06$ & $\ldots$ & $<-2.7 \pm 0.14$ \\
\hline J162724.19-242929.8 & $(1.52 \pm 0.17 \pm 0.08) \times 10^{-1}$ & $>57.1 \pm 4.6$ & $(1.52 \pm 0.19 \pm 0.08) \times 10^{-1}$ & $>33.3 \pm 11.1$ & $-0.0 \pm 0.37$ \\
\hline J162725.40-244659.7 & $(9.60 \pm 1.20 \pm 0.48) \times 10^{-2}$ & $>26.5 \pm 14.4$ & & & \\
\hline J162726.90-244050.8 & $(1.87 \pm 0.06 \pm 0.09) \times 10^{+0}$ & $6.1 \pm 5.5$ & $(1.53 \pm 0.05 \pm 0.08) \times 10^{+0}$ & $20.0 \pm 4.7$ & $-0.41 \pm 0.17$ \\
\hline J162727.36-243116.8 & $(1.76 \pm 0.20 \pm 0.09) \times 10^{-1}$ & $58.6 \pm 12.7$ & $(1.26 \pm 0.15 \pm 0.06) \times 10^{-1}$ & $34.6 \pm 17.9$ & $-0.68 \pm 0.36$ \\
\hline J162728.00-243933.7 & $(3.50 \pm 0.22 \pm 0.17) \times 10^{-1}$ & $22.3 \pm 10.1$ & $(3.96 \pm 0.30 \pm 0.20) \times 10^{-1}$ & $12.3 \pm 12.8$ & $0.25 \pm 0.24$ \\
\hline $\mathrm{J} 162729.23-241755.3$ & $(3.90 \pm 0.06 \pm 0.20) \times 10^{+0}$ & $18.6 \pm 1.3$ & $(2.33 \pm 0.06 \pm 0.12) \times 10^{+0}$ & $28.9 \pm 2.7$ & $-1.04 \pm 0.15$ \\
\hline $\mathrm{J} 162730.82-244727.2$ & $(8.44 \pm 0.16 \pm 0.42) \times 10^{-1}$ & $64.2 \pm 2.2$ & $(4.87 \pm 0.65 \pm 0.24) \times 10^{-1}$ & $>29.7 \pm 7.7$ & $-1.11 \pm 0.31$ \\
\hline $\mathrm{J} 162731.05-243403.4$ & $(1.64 \pm 0.22 \pm 0.08) \times 10^{-1}$ & $>69.3 \pm 2.2$ & $(3.58 \pm 0.29 \pm 0.18) \times 10^{-1}$ & $>89.0 \pm 0.4$ & $1.58 \pm 0.35$ \\
\hline $\mathrm{J} 162731.19-242833.8$ & $(1.87 \pm 0.09 \pm 0.09) \times 10^{-1}$ & $>61.3 \pm 1.2$ & $(1.05 \pm 0.05 \pm 0.05) \times 10^{-1}$ & $51.7 \pm 6.2$ & $-1.17 \pm 0.20$ \\
\hline J162732.68-243324.5 & $(1.84 \pm 0.19 \pm 0.09) \times 10^{-1}$ & $44.7 \pm 13.0$ & $(2.00 \pm 0.15 \pm 0.10) \times 10^{-1}$ & $61.2 \pm 9.1$ & $0.17 \pm 0.29$ \\
\hline $\mathrm{J} 162732.76-241401.7$ & $(3.32 \pm 0.19 \pm 0.17) \times 10^{-1}$ & $58.0 \pm 6.6$ & & $\ldots$ & $\ldots$ \\
\hline $\mathrm{J} 162734.55-242020.7$ & $(1.18 \pm 0.03 \pm 0.06) \times 10^{+0}$ & $29.6 \pm 4.2$ & $(6.72 \pm 0.25 \pm 0.34) \times 10^{-1}$ & $38.7 \pm 6.0$ & $-1.14 \pm 0.17$ \\
\hline $\mathrm{J} 162735.13-242624.0$ & $(3.48 \pm 0.12 \pm 0.17) \times 10^{-1}$ & $17.3 \pm 6.8$ & $(1.72 \pm 0.19 \pm 0.09) \times 10^{-1}$ & $45.8 \pm 9.4$ & $-1.42 \pm 0.27$ \\
\hline J162736.00-241402.9 & $(2.34 \pm 0.24 \pm 0.12) \times 10^{-1}$ & $>58.6 \pm 3.9$ & & & \\
\hline J162738.20-242630.5 & $(2.73 \pm 0.13 \pm 0.14) \times 10^{-1}$ & $12.2 \pm 10.3$ & $(1.98 \pm 0.17 \pm 0.10) \times 10^{-1}$ & $22.8 \pm 12.7$ & $-0.65 \pm 0.24$ \\
\hline J162739.42-243915.8 & $(7.20 \pm 1.50 \pm 0.36) \times 10^{-2}$ & $44.5 \pm 22.6$ & $(1.25 \pm 0.15 \pm 0.06) \times 10^{-1}$ & $>49.3 \pm 11.0$ & $1.11 \pm 0.51$ \\
\hline $\mathrm{J} 162740.92-241452.8$ & $(2.68 \pm 0.39 \pm 0.13) \times 10^{+0}$ & $22.7 \pm 14.7$ & $\ldots$ & $\ldots$ & $\ldots$ \\
\hline J162741.39-241454.5 & $(7.38 \pm 0.34 \pm 0.37) \times 10^{-1}$ & $41.6 \pm 8.2$ & & $\ldots$ & $\ldots$ \\
\hline $\mathrm{J} 162741.49-243537.6$ & $(1.43 \pm 0.15 \pm 0.07) \times 10^{-1}$ & $>61.8 \pm 2.6$ & $(1.02 \pm 0.13 \pm 0.05) \times 10^{-1}$ & $>25.7 \pm 13.1$ & $-0.68 \pm 0.36$ \\
\hline $\mathrm{J} 162742.12-241455.4$ & $(9.70 \pm 1.82 \pm 0.48) \times 10^{-1}$ & $25.4 \pm 15.4$ & $\ldots$ & $\ldots$ & \\
\hline J162745.42-243754.6 & $(8.20 \pm 1.50 \pm 0.41) \times 10^{-2}$ & $>22.7 \pm 15.1$ & $<0.05$ & $\ldots$ & $<-0.84 \pm 0.14$ \\
\hline J162749.85-242540.5 & $(8.00 \pm 0.10 \pm 0.40) \times 10^{+0}$ & $32.3 \pm 2.3$ & $(8.51 \pm 0.27 \pm 0.43) \times 10^{+0}$ & $31.1 \pm 3.1$ & $0.12 \pm 0.16$ \\
\hline $\mathrm{J} 162751.37-242750.4$ & $(2.86 \pm 0.23 \pm 0.14) \times 10^{-1}$ & $>22.1 \pm 6.8$ & $(1.27 \pm 0.15 \pm 0.06) \times 10^{-1}$ & $\ldots{ }^{a}$ & $-1.64 \pm 0.32$ \\
\hline $\mathrm{J} 162751.80-243145.9$ & $(5.98 \pm 1.00 \pm 0.30) \times 10^{-2}$ & $\ldots{ }^{\mathrm{a}}$ & $<0.05$ & $\ldots$ & $<-0.37 \pm 0.14$ \\
\hline J162751.89-244630.1 & $(7.69 \pm 0.42 \pm 0.38) \times 10^{-1}$ & $>95.6 \pm 0.1$ & $(6.61 \pm 0.37 \pm 0.33) \times 10^{-1}$ & $>92.3 \pm 0.4$ & $-0.31 \pm 0.21$ \\
\hline $\mathrm{J} 162752.08-244050.5$ & $(1.67 \pm 0.05 \pm 0.08) \times 10^{+0}$ & $76.1 \pm 1.0$ & $(2.21 \pm 0.07 \pm 0.11) \times 10^{+0}$ & $84.7 \pm 0.6$ & $0.57 \pm 0.17$ \\
\hline J162752.30-242929.9 & $(8.70 \pm 1.40 \pm 0.43) \times 10^{-2}$ & $>43.8 \pm 14.8$ & $(1.05 \pm 0.17 \pm 0.05) \times 10^{-1}$ & $>17.1 \pm 16.9$ & $0.38 \pm 0.48$ \\
\hline $\mathrm{J} 162753.08-242830.3$ & $(2.34 \pm 0.21 \pm 0.12) \times 10^{-1}$ & $48.9 \pm 11.8$ & $(1.74 \pm 0.29 \pm 0.09) \times 10^{-1}$ & $>45.3 \pm 7.5$ & $-0.6 \pm 0.41$ \\
\hline J162756.01-244810.7 & $<0.05$ & $\ldots$ & $(1.09 \pm 0.20 \pm 0.05) \times 10^{-1}$ & $>55.8 \pm 15.9$ & $>1.53 \pm 0.14$ \\
\hline J162757.81-244001.9 & $(3.14 \pm 0.13 \pm 0.16) \times 10^{-1}$ & $61.3 \pm 4.1$ & $(2.62 \pm 0.13 \pm 0.13) \times 10^{-1}$ & $64.7 \pm 6.7$ & $-0.37 \pm 0.19$ \\
\hline $\mathrm{J} 162759.09-242225.9$ & $(2.43 \pm 0.32 \pm 0.12) \times 10^{-1}$ & $21.5 \pm 15.2$ & $(1.34 \pm 0.22 \pm 0.07) \times 10^{-1}$ & $>25.8 \pm 20.8$ & $-1.2 \pm 0.45$ \\
\hline $\mathrm{J} 162759.95-244819.5$ & $(1.96 \pm 0.14 \pm 0.10) \times 10^{+0}$ & $>97.0 \pm 0.1$ & $(2.64 \pm 0.21 \pm 0.13) \times 10^{+0}$ & $>95.9 \pm 0.1$ & $0.6 \pm 0.26$ \\
\hline $\mathrm{J} 162803.39-242124.8$ & $(2.30 \pm 0.22 \pm 0.12) \times 10^{-1}$ & $39.6 \pm 6.7$ & $<0.18$ & $\ldots$ & $<-0.5 \pm 0.14$ \\
\hline $\mathrm{J} 162803.51-242131.2$ & $(1.73 \pm 0.24 \pm 0.09) \times 10^{-1}$ & $>43.2 \pm 5.5$ & $<0.18$ & $\ldots$ & $<0.08 \pm 0.14$ \\
\hline $\mathrm{J} 162804.58-244838.0$ & $(8.80 \pm 1.50 \pm 0.44) \times 10^{-2}$ & $>18.2 \pm 16.4$ & $\ldots$ & $\ldots$ & $\ldots$ \\
\hline $\mathrm{J} 162804.65-243456.6$ & $(2.66 \pm 0.29 \pm 0.13) \times 10^{-1}$ & $>76.6 \pm 1.3$ & $(1.60 \pm 0.24 \pm 0.08) \times 10^{-1}$ & $>58.5 \pm 3.8$ & $-1.03 \pm 0.40$ \\
\hline J162807.21-243040.9 & $(1.39 \pm 0.15 \pm 0.07) \times 10^{-1}$ & $41.2 \pm 26.9$ & $\ldots$ & $\ldots$ & $\ldots$ \\
\hline J162807.28-244201.3 & $(8.50 \pm 1.50 \pm 0.43) \times 10^{-2}$ & $>45.0 \pm 12.8$ & $\ldots$ & $\ldots$ & \\
\hline J162813.72-244008.6 & $(8.49 \pm 0.83 \pm 0.42) \times 10^{-1}$ & $22.3 \pm 13.2$ & $\ldots$ & $\ldots$ & $\ldots$ \\
\hline
\end{tabular}


Table 1

(Continued)

\begin{tabular}{|c|c|c|c|c|c|}
\hline \multirow[t]{2}{*}{ GB-VLA Name } & \multicolumn{4}{|c|}{ Flux Properties } & \multirow[t]{2}{*}{ Spectral Index } \\
\hline & $f_{4.5}(\mathrm{mJy})$ & Var. $(\%)$ & $f_{7.5}(\mathrm{mJy})$ & Var. $(\%)$ & \\
\hline J162820.60-242546.1 & $(1.26 \pm 0.03 \pm 0.06) \times 10^{+1}$ & $35.2 \pm 2.3$ & & $\ldots$ & \\
\hline $\mathrm{J} 163012.16-243345.2$ & $(2.57 \pm 0.06 \pm 0.13) \times 10^{+0}$ & $65.4 \pm 1.3$ & & $\ldots$ & $\ldots$ \\
\hline $\mathrm{J} 163027.69-243300.2$ & $(2.40 \pm 0.29 \pm 0.12) \times 10^{-1}$ & $55.3 \pm 8.2$ & $(2.02 \pm 0.20 \pm 0.10) \times 10^{-1}$ & $45.5 \pm 11.8$ & $-0.35 \pm 0.35$ \\
\hline $\mathrm{J} 163031.02-243158.2$ & $(2.82 \pm 0.56 \pm 0.14) \times 10^{-1}$ & $5.0 \pm 17.0$ & $(1.91 \pm 0.25 \pm 0.10) \times 10^{-1}$ & $>59.4 \pm 4.4$ & $-0.79 \pm 0.50$ \\
\hline $\mathrm{J} 163032.26-243127.9$ & $(7.88 \pm 0.22 \pm 0.39) \times 10^{-1}$ & $42.5 \pm 5.3$ & $(9.22 \pm 0.60 \pm 0.46) \times 10^{-1}$ & $43.5 \pm 6.2$ & $0.32 \pm 0.20$ \\
\hline $\mathrm{J} 163033.26-243038.7$ & $(1.69 \pm 0.28 \pm 0.08) \times 10^{-1}$ & $>60.7 \pm 5.9$ & $(2.20 \pm 0.75 \pm 0.11) \times 10^{-1}$ & $>24.1 \pm 12.1$ & $0.53 \pm 0.78$ \\
\hline $\mathrm{J} 163033.64-243519.0$ & $(1.32 \pm 0.19 \pm 0.07) \times 10^{-1}$ & $>10.6 \pm 24.5$ & $(1.51 \pm 0.16 \pm 0.08) \times 10^{-1}$ & $38.1 \pm 19.6$ & $0.27 \pm 0.39$ \\
\hline $\mathrm{J} 163035.21-243417.8$ & $(1.94 \pm 0.27 \pm 0.10) \times 10^{-1}$ & $21.3 \pm 21.7$ & $(1.33 \pm 0.15 \pm 0.07) \times 10^{-1}$ & $45.2 \pm 11.5$ & $-0.76 \pm 0.39$ \\
\hline $\mathrm{J} 163035.63-243418.9$ & $(1.03 \pm 0.04 \pm 0.05) \times 10^{+0}$ & $33.3 \pm 6.6$ & $(1.07 \pm 0.03 \pm 0.05) \times 10^{+0}$ & $16.6 \pm 8.3$ & $0.08 \pm 0.18$ \\
\hline $\mathrm{J} 163036.26-243135.3$ & $(7.40 \pm 0.19 \pm 0.37) \times 10^{-1}$ & $41.4 \pm 4.7$ & $(5.66 \pm 0.31 \pm 0.28) \times 10^{-1}$ & $36.7 \pm 9.1$ & $-0.54 \pm 0.19$ \\
\hline $\mathrm{J} 163036.93-241334.9$ & $(6.80 \pm 0.44 \pm 0.34) \times 10^{-1}$ & $17.0 \pm 9.7$ & $(4.50 \pm 0.23 \pm 0.23) \times 10^{-1}$ & $32.3 \pm 9.5$ & $-0.83 \pm 0.22$ \\
\hline $\mathrm{J} 163037.85-241206.0$ & $(1.41 \pm 0.03 \pm 0.07) \times 10^{+0}$ & $15.1 \pm 6.2$ & $(8.07 \pm 0.21 \pm 0.40) \times 10^{-1}$ & $18.1 \pm 8.4$ & $-1.13 \pm 0.16$ \\
\hline $\mathrm{J} 163058.02-243441.3$ & $(3.43 \pm 0.38 \pm 0.17) \times 10^{-1}$ & $29.4 \pm 12.7$ & $(3.78 \pm 0.64 \pm 0.19) \times 10^{-1}$ & $>26.3 \pm 8.7$ & $0.2 \pm 0.43$ \\
\hline $\mathrm{J} 163100.40-241640.4$ & $(8.44 \pm 0.42 \pm 0.42) \times 10^{-1}$ & $45.7 \pm 4.5$ & & $\ldots$ & $\ldots$ \\
\hline $\mathrm{J} 163109.63-242554.7$ & $(1.07 \pm 0.03 \pm 0.05) \times 10^{+0}$ & $27.9 \pm 5.9$ & $(9.64 \pm 0.75 \pm 0.48) \times 10^{-1}$ & $>42.0 \pm 6.2$ & $-0.21 \pm 0.22$ \\
\hline $\mathrm{J} 163109.79-243008.4$ & $(1.49 \pm 0.02 \pm 0.07) \times 10^{+0}$ & $21.1 \pm 4.2$ & $(5.81 \pm 0.35 \pm 0.29) \times 10^{-1}$ & $27.1 \pm 7.2$ & $-1.9 \pm 0.19$ \\
\hline $\mathrm{J} 163112.95-242722.6$ & $(4.13 \pm 0.28 \pm 0.21) \times 10^{-1}$ & $17.2 \pm 12.2$ & $(1.99 \pm 0.17 \pm 0.10) \times 10^{-1}$ & $>33.3 \pm 9.5$ & $-1.48 \pm 0.26$ \\
\hline $\mathrm{J} 163115.01-243243.9$ & $(8.20 \pm 0.46 \pm 0.41) \times 10^{-1}$ & $78.9 \pm 2.2$ & $(6.31 \pm 0.36 \pm 0.32) \times 10^{-1}$ & $81.9 \pm 2.1$ & $-0.53 \pm 0.22$ \\
\hline $\mathrm{J} 163115.25-243313.8$ & $(2.10 \pm 0.21 \pm 0.10) \times 10^{-1}$ & $25.6 \pm 13.8$ & $(1.75 \pm 0.19 \pm 0.09) \times 10^{-1}$ & $17.3 \pm 16.4$ & $-0.37 \pm 0.33$ \\
\hline $\mathrm{J} 163115.75-243402.8$ & $(4.57 \pm 0.30 \pm 0.23) \times 10^{-1}$ & $>79.2 \pm 0.7$ & $(4.82 \pm 0.28 \pm 0.24) \times 10^{-1}$ & $>87.3 \pm 1.0$ & $0.11 \pm 0.23$ \\
\hline $\mathrm{J} 163120.14-242928.5$ & $(9.89 \pm 0.09 \pm 0.49) \times 10^{+0}$ & $11.8 \pm 3.5$ & $(6.23 \pm 0.11 \pm 0.31) \times 10^{+0}$ & $11.5 \pm 3.0$ & $-0.93 \pm 0.15$ \\
\hline $\mathrm{J} 163120.18-243001.0$ & $(2.95 \pm 0.27 \pm 0.15) \times 10^{-1}$ & $59.3 \pm 6.5$ & $(2.85 \pm 0.21 \pm 0.14) \times 10^{-1}$ & $57.2 \pm 8.3$ & $-0.07 \pm 0.28$ \\
\hline $\mathrm{J} 163130.62-243351.6$ & $(7.40 \pm 0.33 \pm 0.37) \times 10^{-1}$ & $8.3 \pm 7.6$ & $(5.03 \pm 0.37 \pm 0.25) \times 10^{-1}$ & $50.0 \pm 6.7$ & $-0.78 \pm 0.22$ \\
\hline J163131.09-242719.6 & $(4.30 \pm 0.13 \pm 0.21) \times 10^{+0}$ & $44.2 \pm 3.0$ & $(3.46 \pm 0.11 \pm 0.17) \times 10^{+0}$ & $33.9 \pm 3.2$ & $-0.44 \pm 0.17$ \\
\hline $\mathrm{J} 163138.57-253220.0$ & $(4.87 \pm 0.48 \pm 0.24) \times 10^{-1}$ & $61.3 \pm 6.4$ & & $\ldots$ & \\
\hline $\mathrm{J} 163140.49-245234.7$ & $(7.68 \pm 0.37 \pm 0.38) \times 10^{-1}$ & $38.9 \pm 6.9$ & $(4.67 \pm 0.42 \pm 0.23) \times 10^{-1}$ & $\ldots a$ & $-1.01 \pm 0.25$ \\
\hline $\mathrm{J} 163140.67-241516.4$ & $(9.20 \pm 0.62 \pm 0.46) \times 10^{-1}$ & $71.2 \pm 2.9$ & $<0.47$ & $\ldots$ & $<-1.36 \pm 0.14$ \\
\hline $\mathrm{J} 163151.93-245617.4$ & $(2.87 \pm 0.13 \pm 0.14) \times 10^{+0}$ & $93.3 \pm 0.7$ & $(4.43 \pm 0.31 \pm 0.22) \times 10^{+0}$ & $97.5 \pm 0.4$ & $0.88 \pm 0.22$ \\
\hline $\mathrm{J} 163152.10-245615.7$ & $(3.63 \pm 0.37 \pm 0.18) \times 10^{-1}$ & $>67.2 \pm 5.4$ & $(1.26 \pm 0.09 \pm 0.06) \times 10^{+0}$ & $>41.1 \pm 4.8$ & $2.52 \pm 0.29$ \\
\hline $\mathrm{J} 163152.34-253144.7$ & $(1.50 \pm 0.19 \pm 0.07) \times 10^{-1}$ & $16.5 \pm 19.1$ & $(1.12 \pm 0.22 \pm 0.06) \times 10^{-1}$ & $>34.8 \pm 8.8$ & $-0.59 \pm 0.49$ \\
\hline $\mathrm{J} 163154.49-245217.1$ & $(6.46 \pm 0.17 \pm 0.32) \times 10^{-1}$ & $35.5 \pm 4.7$ & $(3.53 \pm 0.27 \pm 0.18) \times 10^{-1}$ & $30.8 \pm 15.4$ & $-1.22 \pm 0.22$ \\
\hline $\mathrm{J} 163159.36-245639.7$ & $(2.19 \pm 0.04 \pm 0.11) \times 10^{+0}$ & $10.8 \pm 5.2$ & $(1.56 \pm 0.07 \pm 0.08) \times 10^{+0}$ & $26.1 \pm 6.2$ & $-0.69 \pm 0.17$ \\
\hline $\mathrm{J} 163159.51-252918.7$ & $(3.28 \pm 0.21 \pm 0.16) \times 10^{-1}$ & $23.7 \pm 11.9$ & $(2.52 \pm 0.17 \pm 0.13) \times 10^{-1}$ & $5.6 \pm 17.5$ & $-0.53 \pm 0.24$ \\
\hline $\mathrm{J} 163200.97-245643.3$ & $(7.19 \pm 0.37 \pm 0.36) \times 10^{-1}$ & $52.7 \pm 5.8$ & $(7.94 \pm 0.27 \pm 0.40) \times 10^{-1}$ & $43.7 \pm 3.6$ & $0.2 \pm 0.19$ \\
\hline $\mathrm{J} 163202.39-245710.0$ & $(2.86 \pm 0.40 \pm 0.14) \times 10^{-1}$ & $>67.4 \pm 3.6$ & $(2.15 \pm 0.39 \pm 0.11) \times 10^{-1}$ & $>42.5 \pm 5.3$ & $-0.58 \pm 0.48$ \\
\hline $\mathrm{J} 163204.79-245636.8$ & $(4.35 \pm 0.53 \pm 0.22) \times 10^{-1}$ & $34.3 \pm 12.4$ & $<0.12$ & $\ldots$ & $<-2.6 \pm 0.14$ \\
\hline $\mathrm{J} 163208.05-253016.3$ & $(6.08 \pm 0.08 \pm 0.30) \times 10^{+0}$ & $2.2 \pm 5.9$ & $(4.11 \pm 0.09 \pm 0.21) \times 10^{+0}$ & $5.9 \pm 8.1$ & $-0.79 \pm 0.15$ \\
\hline J163210.20-245618.9 & $(3.12 \pm 0.41 \pm 0.16) \times 10^{-1}$ & $>44.3 \pm 5.5$ & $(2.04 \pm 0.13 \pm 0.10) \times 10^{-1}$ & $\begin{array}{l}\ldots^{\mathrm{a}} \\
\mathrm{a}^{0.1}\end{array}$ & $-0.86 \pm 0.33$ \\
\hline $\mathrm{J} 163210.77-243827.6$ & $(5.33 \pm 0.33 \pm 0.27) \times 10^{-1}$ & $23.1 \pm 7.7$ & $(3.61 \pm 0.27 \pm 0.18) \times 10^{-1}$ & $57.6 \pm 9.2$ & $-0.79 \pm 0.24$ \\
\hline $\mathrm{J} 163210.96-253021.3$ & $(1.23 \pm 0.16 \pm 0.06) \times 10^{+0}$ & $\ldots$ & $<0.13$ & $\ldots$ & Extended \\
\hline $\mathrm{J} 163211.08-243651.1$ & $(7.69 \pm 0.27 \pm 0.38) \times 10^{-1}$ & $38.7 \pm 5.9$ & $(6.62 \pm 0.82 \pm 0.33) \times 10^{-1}$ & $>25.5 \pm 14.9$ & $-0.3 \pm 0.30$ \\
\hline $\mathrm{J} 163211.79-244021.8$ & $(1.50 \pm 0.03 \pm 0.08) \times 10^{+0}$ & $28.0 \pm 3.4$ & $(1.32 \pm 0.05 \pm 0.07) \times 10^{+0}$ & $30.0 \pm 6.8$ & $-0.27 \pm 0.16$ \\
\hline $\mathrm{J} 163212.25-243643.7$ & $(6.03 \pm 0.22 \pm 0.30) \times 10^{-1}$ & $28.7 \pm 8.0$ & $<0.26$ & $\ldots$ & $<-1.7 \pm 0.14$ \\
\hline $\mathrm{J} 163213.92-244407.8$ & $(3.37 \pm 0.53 \pm 0.17) \times 10^{-1}$ & $>54.5 \pm 7.6$ & $\ldots$ & $\ldots$ & $\ldots$ \\
\hline $\mathrm{J} 163214.16-252344.5$ & $(2.44 \pm 0.06 \pm 0.12) \times 10^{+1}$ & $29.2 \pm 1.7$ & $\ldots$ & $\cdots$ & $\ldots$ \\
\hline $\mathrm{J} 163227.41-243951.4$ & $(6.82 \pm 0.25 \pm 0.34) \times 10^{-1}$ & $20.4 \pm 5.1$ & $(4.45 \pm 0.21 \pm 0.22) \times 10^{-1}$ & $37.2 \pm 5.8$ & $-0.86 \pm 0.19$ \\
\hline $\mathrm{J} 163231.17-244014.6$ & $(5.10 \pm 0.27 \pm 0.26) \times 10^{-1}$ & $34.3 \pm 5.9$ & $(4.16 \pm 0.25 \pm 0.21) \times 10^{-1}$ & $32.5 \pm 6.6$ & $-0.41 \pm 0.22$ \\
\hline $\mathrm{J} 163245.23-243647.4$ & $(7.08 \pm 0.57 \pm 0.35) \times 10^{-1}$ & $51.0 \pm 7.8$ & & $\ldots$ & $\ldots$ \\
\hline $\mathrm{J} 163421.10-235625.1$ & $(1.80 \pm 0.20 \pm 0.09) \times 10^{-1}$ & $>76.1 \pm 1.2$ & $(1.48 \pm 0.16 \pm 0.07) \times 10^{-1}$ & $>73.7 \pm 2.5$ & $-0.4 \pm 0.34$ \\
\hline J163436.01-235614.5 & $(8.57 \pm 0.56 \pm 0.43) \times 10^{-1}$ & $19.7 \pm 9.5$ & $(4.72 \pm 0.77 \pm 0.24) \times 10^{-1}$ & $33.2 \pm 12.5$ & $-1.21 \pm 0.38$ \\
\hline $\mathrm{J} 163437.30-235946.2$ & $(5.88 \pm 0.34 \pm 0.29) \times 10^{-1}$ & $17.5 \pm 8.2$ & $(7.37 \pm 0.56 \pm 0.37) \times 10^{-1}$ & $>33.1 \pm 8.4$ & $0.46 \pm 0.24$ \\
\hline $\mathrm{J} 163551.89-242253.6$ & $(5.44 \pm 0.50 \pm 0.27) \times 10^{-1}$ & $45.5 \pm 7.5$ & …… & a & $\ldots$ \\
\hline J163557.74-241447.9 & $(1.77 \pm 0.06 \pm 0.09) \times 10^{+0}$ & $32.8 \pm 8.5$ & & $\ldots$ & \\
\hline $\mathrm{J} 163615.79-242159.8$ & $(2.61 \pm 0.36 \pm 0.13) \times 10^{-1}$ & $15.3 \pm 10.6$ & $(2.12 \pm 0.14 \pm 0.11) \times 10^{-1}$ & $7.9 \pm 14.2$ & $-0.42 \pm 0.34$ \\
\hline $\mathrm{J} 163617.50-242555.4$ & $(2.06 \pm 0.07 \pm 0.10) \times 10^{+0}$ & $11.4 \pm 7.0$ & $(1.47 \pm 0.17 \pm 0.07) \times 10^{+0}$ & $>29.7 \pm 7.4$ & $-0.68 \pm 0.28$ \\
\hline $\mathrm{J} 163626.93-242117.8$ & $(1.76 \pm 0.20 \pm 0.09) \times 10^{-1}$ & $29.8 \pm 15.7$ & $(2.00 \pm 0.31 \pm 0.10) \times 10^{-1}$ & $>35.9 \pm 10.4$ & $0.26 \pm 0.41$ \\
\hline $\mathrm{J} 163639.40-241710.3$ & $(1.26 \pm 0.06 \pm 0.06) \times 10^{+0}$ & $46.0 \pm 6.1$ & & $\ldots$ & $\ldots$ \\
\hline $\mathrm{J} 163949.54-235939.0$ & $(2.62 \pm 0.41 \pm 0.13) \times 10^{-1}$ & $32.9 \pm 19.9$ & $(1.90 \pm 0.27 \pm 0.10) \times 10^{-1}$ & $>25.2 \pm 8.0$ & $-0.65 \pm 0.45$ \\
\hline $\mathrm{J} 164002.06-240137.0$ & $(3.96 \pm 0.26 \pm 0.20) \times 10^{-1}$ & $19.2 \pm 10.5$ & $(2.15 \pm 0.24 \pm 0.11) \times 10^{-1}$ & $>10.3 \pm 14.1$ & $-1.23 \pm 0.30$ \\
\hline
\end{tabular}

Note. ${ }^{\text {a }}$ Source not detected at three times the noise level on individual epochs, but detected on the image of the concatenated epochs. 


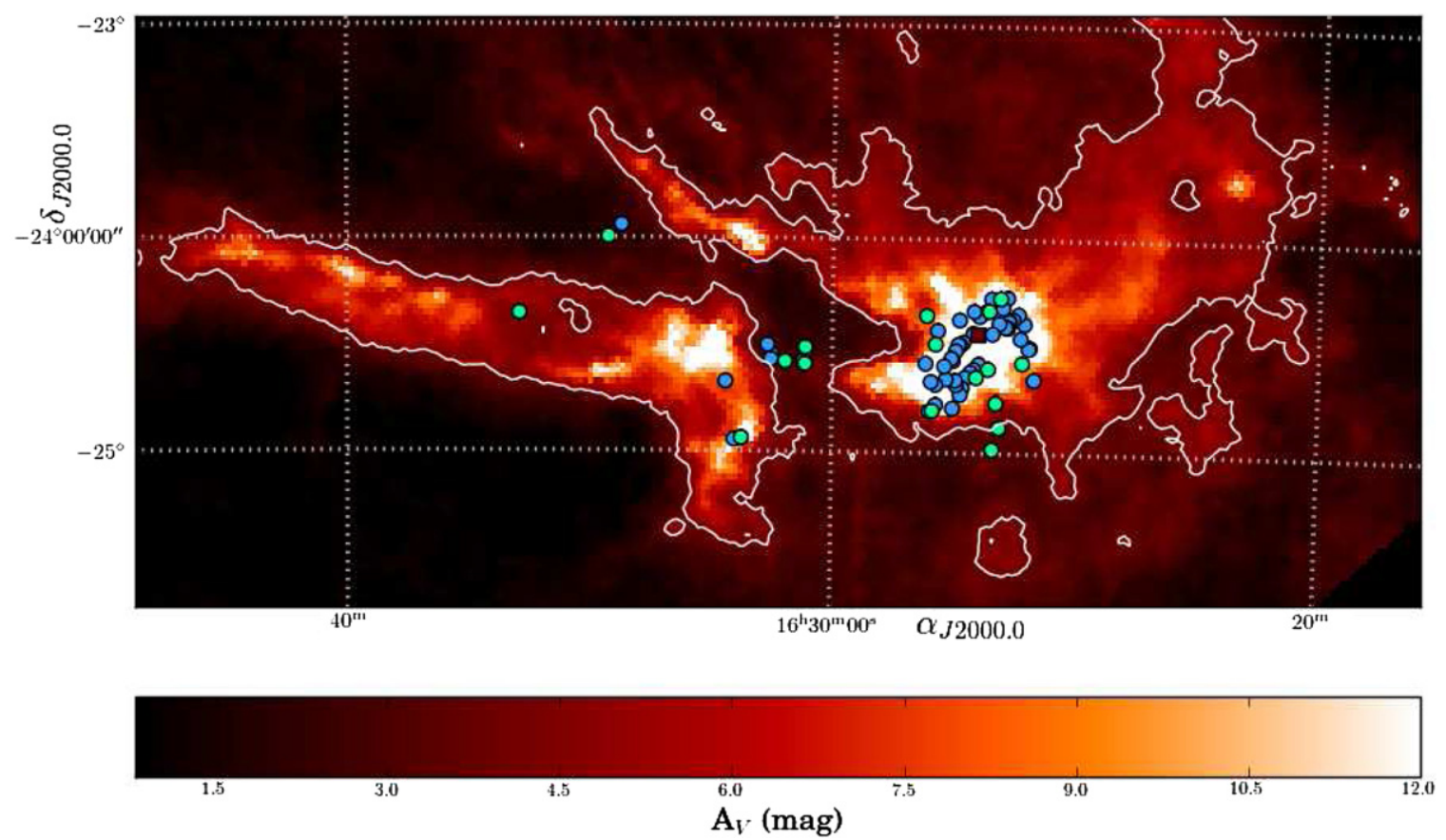

Figure 2. Location of the YSO (blue circles) and candidate YSO (green circle) onto the extinction map of the Ophiuchus complex. The position of the new calibrator detected here (GBS-VLA J162700.00-242640.3) is indicated as a brown square in the Ophiuchus core.

(A color version of this figure is available in the online journal.)

Table 2

Sources Detected in Circular Polarization

\begin{tabular}{lcrc}
\hline \hline GB-VLA Name & Source Type & $\begin{array}{c}\text { X Polz } \\
(\%)\end{array}$ & $\begin{array}{c}\text { C Polz } \\
(\%)\end{array}$ \\
\hline J162557.51-243032.1 & YSO/III & $22.1(\mathrm{~L})$ & $<16.3$ \\
J162603.01-242336.4 & YSO/III & $6.5(\mathrm{~L})$ & $4.7(\mathrm{~L})$ \\
J162634.17-242328.4 & YSO/III & $5.8(\mathrm{~L})$ & $\ldots$ \\
J162752.08-244050.5 & YSO & $11.2(\mathrm{~L})$ & $8.9(\mathrm{~L})$ \\
J163115.01-243243.9 & YSO/WTT & $16.2(\mathrm{~L})$ & $8.3(\mathrm{~L})$ \\
J163200.97-245643.3 & YSO/I & $21.4(\mathrm{R})$ & $16.1(\mathrm{R})$ \\
J163211.79-244021.8 & YSO/WTT/II & $\ldots$ & $16.5(\mathrm{~L})$ \\
\hline
\end{tabular}

account in our counterpart search. We considered a radio source associated with a counterpart at another wavelengths if the separation between the two was below the combined uncertainties of the two data sets. This was about $1^{\prime \prime} .5$ for the optical and infrared catalogs, but could be significantly larger for some of the radio catalogs (for instance, the NVSS has a positional uncertainty of about $5^{\prime \prime}$ ). We found that only 76 of the sources detected here had previously been reported at radio wavelengths (Column 7 of Table 3), while the other 113 are new radio detections. On the other hand, we found a total of 100 counterparts at other wavelengths. Note that there are a significant number of sources that were previously known at radio wavelengths and have known counterparts at other frequencies. As a consequence, the number of sources that were previously known (at any frequency) is 134, while 55 of the sources in our sample are reported here for the first time.

Based on their optical/infrared characteristics, 2 of the 100 sources with counterparts are classified in the literature as extragalactic sources, while 55 are classified as YSOs (see Column 8 of Table 3). One additional object (GBS-VLA J162626.31-242430.3, also known as VLA 1623B) is associated with the well-known Class 0 source VLA 1623, but it is still debated whether it is an outflow knot feature, or a protostellar object (see the discussion by Ward-Thompson et al. 2011; and Section 5.1 below). The remaining 42 radio sources with known counterparts at other wavelengths are, to our knowledge, not classified in the literature. On the other hand, two sources (GBS-VLA J162615.67-243421.2 and GBSVLA J162626.03-244923.7) have been classified in the literature as extragalactic, on the basis of their radio properties alone.

To summarize, a total 134 of our 189 sources were previously known (76 at radio wavelengths, and 100 at other frequencies, with some overlap between the two sub-samples). Of these 134 sources, 56 are classified as YSOs and 4 as extragalactic; the other 74 are not classified. Given the existing deep nearand mid-infrared observations of Ophiuchus, it is unlikely that a major population exists of unidentified young stars. In consequence, we argue that most of these unclassified sources are extragalactic. For the same reason, most of the 55 objects detected here for the first time are likely background sources. We note, however, that 18 of the 129 unclassified objects (55 identified here for the first time and 74 previously known at radio wavelengths) are compact, have a positive spectral index, or exhibit high variability (Table 4). Since these latter two properties are not expected of quasars (which are certainly variable, but usually not strongly on such short timescale; e.g., Hovatta et al. 2008), but would be natural characteristics of young stars, we argue that a small population of YSO might be present among the unclassified sources. This population could account for, at most, $15 \%$ of the unclassified sources, and possibly significantly less. The distribution of both the YSO and candidate YSO in our radio sources are shown in Figure 2.

It is interesting that 35 of the sources reported here are only detected at radio frequencies and with Spitzer (with no detection at any other wavelengths). These sources are usually not classified, and might be either young stellar sources, or foreground/background objects. It will be useful to study this population further. 
Table 3

Radio Sources with Known Counterparts

\begin{tabular}{|c|c|c|c|c|c|c|c|}
\hline \multirow[t]{2}{*}{ GB-VLA Name } & \multirow[t]{2}{*}{ Other Names } & \multirow[b]{2}{*}{ X-Ray ${ }^{\mathrm{a}}$} & \multicolumn{3}{|c|}{ Infrared $^{\mathrm{b}, \mathrm{c}}$} & \multirow[t]{2}{*}{ Radio $^{\mathrm{d}}$} & \multirow[t]{2}{*}{$\overline{\text { Object Type }}$} \\
\hline & & & $S S T$ & $2 \mathrm{M}$ & WISE & & \\
\hline J162540.94-244147.2 & SSTc2d J162540.9-244147 & $\ldots$ & $\mathrm{Y}$ & $\ldots$ & $\ldots$ & $\ldots$ & $\ldots$ \\
\hline J162542.48-242143.7 & & $\ldots$ & $\ldots$ & $\ldots$ & $\ldots$ & ROC 4 & $\ldots$ \\
\hline J162548.96-244049.5 & SSTc2d J162549.0-244049 & $\ldots$ & $\mathrm{Y}$ & $\ldots$ & $\ldots$ & ROC 6 & $\ldots$ \\
\hline J162550.51-243914.7 & WLY 2-10 & ROXRA 5 & $\mathrm{Y}$ & $\mathrm{Y}$ & $\mathrm{Y}$ & $\ldots$ & YSO \\
\hline J162556.09-243015.3 & WLY 2-11 & $\ldots$ & $\mathrm{Y}$ & $\mathrm{Y}$ & $\mathrm{Y}$ & GDS J162556.1-243014 & YSO \\
\hline J162557.51-243032.1 & YLW 24 & ROXRA 7 & $\mathrm{Y}$ & $\mathrm{Y}$ & $\mathrm{Y}$ & & YSO \\
\hline J162603.01-242336.4 & DoAr 21 & A-2 & $\mathrm{Y}$ & $\mathrm{Y}$ & $\mathrm{Y}$ & ROC 8 & YSO \\
\hline J162607.63-242741.7 & BKLT J162607-242742 & A-5 & $\mathrm{Y}$ & $\mathrm{Y}$ & $\mathrm{Y}$ & $\ldots$ & YSO \\
\hline J162608.04-242523.1 & & $\ldots$ & $\ldots$ & $\ldots$ & $\ldots$ & ROC 9 & $\ldots$ \\
\hline J162610.32-242054.9 & GSS 26 & A-6 & $\mathrm{Y}$ & $\mathrm{Y}$ & $\mathrm{Y}$ & GDS J162610.3-242054 & YSO \\
\hline J162610.55-242853.3 & $\ldots$ & $\ldots$ & $\ldots$ & $\ldots$ & $\ldots$ & GDS J162610.5-242853 & $\ldots$ \\
\hline J162611.08-242907.4 & GDS J162611.0-242908 & $\ldots$ & $\ldots$ & $\ldots$ & $\ldots$ & GDS J162611.0-242908 & $\ldots$ \\
\hline $\mathrm{J} 162615.67-243421.2$ & FG Oph 18 & $\ldots$ & $\ldots$ & $\ldots$ & $\ldots$ & NVSS 162615-243419 & $\mathrm{E}$ \\
\hline J162616.84-242223.5 & BKLT J162616-242225 & A-14 & $\mathrm{Y}$ & $\mathrm{Y}$ & $\mathrm{Y}$ & LFAM p1 & YSO \\
\hline $\mathrm{J} 162621.72-242250.7$ & SSTc2d J162621.7-242250 & $\ldots$ & $\mathrm{Y}$ & $\ldots$ & $\ldots$ & LFAM 1 & YSO \\
\hline J162622.38-242253.3 & BKLT J162622-242254 & A-20 & $\mathrm{Y}$ & $\mathrm{Y}$ & $\ldots$ & LFAM 2 & YSO \\
\hline J162623.57-242439.6 & BKLT J162623-242441 & A-23 & $\mathrm{Y}$ & $\mathrm{Y}$ & $\mathrm{Y}$ & LFAM 3 & YSO \\
\hline J162624.08-241613.5 & YLW 32 & A-25 & $\mathrm{Y}$ & $\mathrm{Y}$ & $\mathrm{Y}$ & GDS J162624.0-241613 & YSO \\
\hline J162625.62-242429.2 & VLA $1623 \mathrm{~W}$ & $\ldots$ & $\mathrm{Y}$ & $\ldots$ & $\ldots$ & LFAM 4 & YSO \\
\hline J162626.03-244923.7 & SFAM 11 & $\ldots$ & $\mathrm{Y}$ & $\ldots$ & $\ldots$ & $\mathrm{ROC} 12$ & $\mathrm{E}$ \\
\hline J162626.31-242430.2 & VLA 1623B & $\ldots$ & $\ldots$ & $\ldots$ & $\ldots$ & (?) Knot of VLA 1623 & YSO? \\
\hline J162626.39-242430.9 & VLA $1623 \mathrm{~A}$ & $\ldots$ & $\mathrm{Y}$ & $\ldots$ & $\ldots$ & VLA 1623 & YSO \\
\hline J162629.63-242317.2 & $\ldots$ & $\ldots$ & $\ldots$ & $\ldots$ & $\ldots$ & GDS J162629.5-242317 & $\ldots$ \\
\hline J162629.67-241905.8 & BKLT J162629-241908 & A-33 & $\mathrm{Y}$ & $\mathrm{Y}$ & $\mathrm{Y}$ & LFAM 8 & YSO \\
\hline J162630.59-242023.0 & GDS J162630.6-242023 & A-35 & $\ldots$ & $\ldots$ & $\ldots$ & $\ldots$ & $\ldots$ \\
\hline J162631.28-241832.9 & GDS J162631.3-241833 & A-37 & $\ldots$ & $\ldots$ & $\ldots$ & LFAM 10 & $\ldots$ \\
\hline J162633.48-241215.9 & GDS J162633.4-241216 & $\ldots$ & $\ldots$ & $\ldots$ & $\ldots$ & SFAM 12 & $\ldots$ \\
\hline J162634.17-242328.4 & S1 & A-41 & $\mathrm{Y}$ & $\mathrm{Y}$ & $\ldots$ & SFAM 13 & YSO \\
\hline J162634.95-242655.3 & $\ldots$ & $\ldots$ & $\ldots$ & $\ldots$ & $\ldots$ & LFAM 12 & $\ldots$ \\
\hline J162635.33-242405.2 & GDS J162635.3-242405 & $\ldots$ & $\ldots$ & $\ldots$ & $\ldots$ & LFAM 13 & $\ldots$ \\
\hline J162639.00-243052.8 & $\ldots$ & $\ldots$ & $\ldots$ & $\ldots$ & $\ldots$ & GDS J162639.0-243052 & $\ldots$ \\
\hline J162642.44-242626.1 & BKLT J162642-242627 & A-51 & $\mathrm{Y}$ & $\mathrm{Y}$ & $\mathrm{Y}$ & LFAM 15 & YSO \\
\hline J162642.53-244628.3 & SSTc2d J162642.5-244628 & $\ldots$ & $\mathrm{Y}$ & $\ldots$ & $\ldots$ & $\ldots$ & $\ldots$ \\
\hline J162643.76-241633.4 & VSSG 11 & A-56 & $\mathrm{Y}$ & $\mathrm{Y}$ & $\mathrm{Y}$ & SFAM 15 & YSO \\
\hline $\mathrm{J} 162646.36-242002.0$ & GDS J162646.3-242001 & $\ldots$ & $\ldots$ & $\ldots$ & $\ldots$ & LFAM 17 & $\ldots$ \\
\hline J162647.17-245157.4 & SSTc2d J162647.2-245157 & $\ldots$ & $\mathrm{Y}$ & $\ldots$ & $\ldots$ & $\ldots$ & $\ldots$ \\
\hline J162649.23-242003.3 & VSSG 3 & A- 63 & $\mathrm{Y}$ & $\mathrm{Y}$ & $\mathrm{Y}$ & LFAM 18 & YSO \\
\hline J162651.69-241441.5 & SSTc2d J162651.7-241441 & $\ldots$ & $\mathrm{Y}$ & $\mathrm{Y}$ & $\mathrm{Y}$ & $\ldots$ & $\ldots$ \\
\hline J162653.38-241105.8 & SSTc2d J162653.4-241106 & $\ldots$ & $\mathrm{Y}$ & $\ldots$ & $\ldots$ & $\ldots$ & $\ldots$ \\
\hline J162658.25-243738.5 & BKLT J162658-243739A & $\ldots$ & $\ldots$ & $\ldots$ & $\ldots$ & $\ldots$ & $\ldots$ \\
\hline J162658.38-242130.5 & YLW 1C & A-72 & $\mathrm{Y}$ & $\mathrm{Y}$ & $\mathrm{Y}$ & $\ldots$ & YSO \\
\hline J162659.16-243458.9 & YLW 4C & DROXO 27 & $\mathrm{Y}$ & $\ldots$ & $\ldots$ & LFAM 23 & YSO \\
\hline $\mathrm{J} 162700.00-242640.3$ & FG Oph 21 & A-75 & $\mathrm{Y}$ & $\ldots$ & $\ldots$ & LFAM 21 & $\mathrm{E}$ \\
\hline J162700.02-243537.6 & $\ldots$ & $\ldots$ & $\ldots$ & $\ldots$ & $\ldots$ & LFAM 24 & $\ldots$ \\
\hline J162702.11-243842.5 & $\ldots$ & $\ldots$ & $\ldots$ & $\ldots$ & $\ldots$ & LFAM 25 & $\ldots$ \\
\hline J162702.15-241927.8 & GDS J162702.1-241928 & $\ldots$ & $\ldots$ & $\ldots$ & $\ldots$ & GDS J162702.1-241928 & $\ldots$ \\
\hline J162702.33-243727.3 & YLW 5 & BF-12 & $\mathrm{Y}$ & $\ldots$ & $\ldots$ & $\ldots$ & YSO \\
\hline $\mathrm{J} 162702.36-242724.8$ & SSTc2d J162702.4-242725 & $\ldots$ & $\mathrm{Y}$ & $\ldots$ & $\ldots$ & GDS J162702.3-242724 & $\ldots$ \\
\hline J162705.16-242007.8 & VSSG 21 & A- 80 & $\mathrm{Y}$ & $\mathrm{Y}$ & $\mathrm{Y}$ & $\ldots$ & YSO \\
\hline J162705.25-243629.8 & ISO-Oph 99 & $\ldots$ & $\mathrm{Y}$ & $\mathrm{Y}$ & $\mathrm{Y}$ & LFAM 26 & YSO \\
\hline J162705.96-242618.9 & YLW 10A & A-81 & $\mathrm{Y}$ & $\mathrm{Y}$ & $\mathrm{Y}$ & $\ldots$ & YSO \\
\hline J162709.41-243719.0 & YLW 7 & DROXO 38 & $\mathrm{Y}$ & $\mathrm{Y}$ & $\mathrm{Y}$ & LFAM 27 & YSO \\
\hline J162711.29-243722.5 & & $\ldots$ & $\ldots$ & $\ldots$ & $\ldots$ & LFAM 28 & $\ldots$ \\
\hline J162713.62-242226.1 & SSTc2d J162713.6-242226 & $\ldots$ & $\mathrm{Y}$ & $\ldots$ & $\ldots$ & LFAM 32 & $\ldots$ \\
\hline J162714.71-243919.7 & $\ldots$ & $\ldots$ & $\ldots$ & $\ldots$ & $\ldots$ & LFAM 29 & $\ldots$ \\
\hline J162715.69-243845.7 & WL 20S & $\ldots$ & $\mathrm{Y}$ & $\mathrm{Y}$ & $\mathrm{Y}$ & $\ldots$ & YSO \\
\hline J162717.38-243616.7 & $\ldots$ & $\ldots$ & $\ldots$ & $\ldots$ & $\ldots$ & LFAM 32 & $\ldots$ \\
\hline J162718.17-242852.9 & YLW 12B & DROXO 49 & $\mathrm{Y}$ & $\mathrm{Y}$ & $\ldots$ & SFAM 22 & YSO \\
\hline J162719.34-243130.4 & & BF-45 & $\ldots$ & $\ldots$ & $\ldots$ & $\ldots$ & $\ldots$ \\
\hline J162719.50-244140.3 & V2247 Oph & DROXO 53 & $\mathrm{Y}$ & $\mathrm{Y}$ & $\mathrm{Y}$ & LFAM p6 & YSO \\
\hline J162721.81-244335.9 & [GY92] 253 & DROXO 55 & $\mathrm{Y}$ & $\mathrm{Y}$ & $\mathrm{Y}$ & $\ldots$ & YSO \\
\hline J162721.97-242940.0 & [GY92] 256 & BF-51 & $\mathrm{Y}$ & $\mathrm{Y}$ & $\ldots$ & $\ldots$ & YSO \\
\hline J162722.96-242236.6 & [MPK2010b] 3809 & $\ldots$ & $\mathrm{Y}$ & $\mathrm{Y}$ & $\ldots$ & $\ldots$ & YSO \\
\hline J162724.19-242929.8 & BKLT J162724-242929 & BF-55 & $\mathrm{Y}$ & $\mathrm{Y}$ & $\ldots$ & $\ldots$ & YSO \\
\hline
\end{tabular}


Table 3

(Continued)

\begin{tabular}{|c|c|c|c|c|c|c|c|}
\hline \multirow[t]{2}{*}{ GB-VLA Name } & \multirow[t]{2}{*}{ Other Names } & \multirow[b]{2}{*}{ X-Ray ${ }^{\mathrm{a}}$} & \multicolumn{3}{|c|}{ Infrared $^{\mathrm{b}, \mathrm{c}}$} & \multirow[t]{2}{*}{ Radio $^{\mathrm{d}}$} & \multirow[t]{2}{*}{ Object Type } \\
\hline & & & $S S T$ & $2 \mathrm{M}$ & WISE & & \\
\hline J162726.90-244050.8 & YLW 15 & DROXO 62 & $\mathrm{Y}$ & $\mathrm{Y}$ & $\mathrm{Y}$ & LFAM 33 & YSO \\
\hline J162727.36-243116.8 & BKLT J162727-243116 & DROXO 63 & $\mathrm{Y}$ & $\mathrm{Y}$ & $\mathrm{Y}$ & $\ldots$ & YSO \\
\hline J162728.00-243933.7 & YLW 16A & DROXO 64 & $\mathrm{Y}$ & $\mathrm{Y}$ & $\mathrm{Y}$ & LFAM 35 & YSO \\
\hline J162729.23-241755.3 & & & & & & ROC 25 & \\
\hline J162730.82-244727.2 & BKLT J162730-244726 & DROXO 71 & $\mathrm{Y}$ & $\mathrm{Y}$ & $\mathrm{Y}$ & $\ldots$ & YSO \\
\hline J162731.05-243403.4 & BKLT J162731-243402 & DROXO 72 & $\mathrm{Y}$ & $\mathrm{Y}$ & $\mathrm{Y}$ & $\ldots$ & YSO \\
\hline J162731.19-242833.8 & OphB2 S7 & $\ldots$ & $\mathrm{Y}$ & $\ldots$ & $\ldots$ & $\ldots$ & $\ldots$ \\
\hline J162732.68-243324.5 & BKLT J162732-243323 & DROXO 75 & $\ldots$ & $\mathrm{Y}$ & $\mathrm{Y}$ & LFAM p7 & YSO \\
\hline J162734.55-242020.7 & $\ldots$ & $\ldots$ & $\ldots$ & $\ldots$ & $\ldots$ & ROC 26 & $\ldots$ \\
\hline J162735.13-242624.0 & $\ldots$ & $\ldots$ & $\ldots$ & $\ldots$ & $\ldots$ & OphB2 S8 & $\ldots$ \\
\hline J162736.00-241402.9 & $\ldots$ & $\ldots$ & $\ldots$ & $\ldots$ & $\ldots$ & ROC 27 & $\ldots$ \\
\hline J162738.20-242630.5 & $\ldots$ & $\ldots$ & $\ldots$ & $\ldots$ & $\ldots$ & OphB2 S9 & $\ldots$ \\
\hline J162739.42-243915.8 & BKLT J162739-243914 & DROXO 86 & $\mathrm{Y}$ & $\mathrm{Y}$ & $\mathrm{Y}$ & LFAM p8 & YSO \\
\hline J162740.92-241452.8 & $\ldots$ & $\ldots$ & $\ldots$ & $\ldots$ & $\ldots$ & ROC 28 & $\ldots$ \\
\hline J162741.39-241454.5 & $\ldots$ & $\ldots$ & $\ldots$ & $\ldots$ & $\ldots$ & ROC 28 & $\ldots$ \\
\hline J162741.49-243537.6 & BKLT J162741-243537 & DROXO 89 & $\mathrm{Y}$ & $\ldots$ & $\ldots$ & $\ldots$ & YSO \\
\hline J162749.85-242540.5 & YLW 53 & $\ldots$ & $\mathrm{Y}$ & $\mathrm{Y}$ & $\mathrm{Y}$ & ROC 31 & YSO \\
\hline J162751.80-243145.9 & YLW 52 & DROXO 97 & $\mathrm{Y}$ & $\mathrm{Y}$ & $\mathrm{Y}$ & $\ldots$ & YSO \\
\hline J162751.89-244630.1 & BKLT J162752-244630 & DROXO 98 & $\mathrm{Y}$ & Y & $\mathrm{Y}$ & $\ldots$ & YSO \\
\hline J162752.08-244050.5 & HBC 642 & DROXO 99 & $\mathrm{Y}$ & Y & $\mathrm{Y}$ & $\ldots$ & YSO \\
\hline J162752.30-242929.9 & SSTc2d J162752.3-242930 & $\ldots$ & $\mathrm{Y}$ & $\ldots$ & $\ldots$ & $\ldots$ & $\ldots$ \\
\hline J162753.08-242830.3 & SSTc2d J162753.1-242830 & $\ldots$ & $\mathrm{Y}$ & $\ldots$ & $\mathrm{Y}$ & $\ldots$ & $\ldots$ \\
\hline J162757.81-244001.9 & BKLT J162757-244004 & DROXO 101 & $\mathrm{Y}$ & $\mathrm{Y}$ & $\mathrm{Y}$ & $\ldots$ & YSO \\
\hline J162759.09-242225.9 & SSTc2d J162759.1-242226 & $\ldots$ & $\mathrm{Y}$ & $\ldots$ & $\ldots$ & $\ldots$ & $\ldots$ \\
\hline J162759.95-244819.5 & BKLT J162800-244819 & DROXO 102 & $\mathrm{Y}$ & Y & $\mathrm{Y}$ & $\ldots$ & YSO \\
\hline J162803.39-242124.8 & ROC 33 & $\ldots$ & $\mathrm{Y}$ & $\ldots$ & $\ldots$ & ROS 15 & $\ldots$ \\
\hline J162803.51-242131.2 & & $\ldots$ & $\ldots$ & $\ldots$ & $\ldots$ & SFAM 30 & $\ldots$ \\
\hline J162804.65-243456.6 & BKLT J162804-243459 & DROXO 105 & $\mathrm{Y}$ & Y & $\mathrm{Y}$ & $\ldots$ & YSO \\
\hline J162807.21-243040.9 & SSTc2d J162807.2-243041 & $\ldots$ & $\mathrm{Y}$ & $\ldots$ & $\ldots$ & $\ldots$ & $\ldots$ \\
\hline J162813.72-244008.6 & SFAM 34 & $\ldots$ & $\ldots$ & $\ldots$ & $\ldots$ & ROC 35 & $\ldots$ \\
\hline J162820.60-242546.1 & SSTc2d J162820.6-242546 & $\ldots$ & $\mathrm{Y}$ & $\ldots$ & $\mathrm{Y}$ & ROC 37 & $\ldots$ \\
\hline J163012.16-243345.2 & SSTc2d J163012.2-243345 & $\ldots$ & $\mathrm{Y}$ & $\ldots$ & $\ldots$ & ROC 43 & $\ldots$ \\
\hline J163027.69-243300.2 & SSTc2d J163027.7-243300 & $\ldots$ & Y & $\ldots$ & $\ldots$ & $\ldots$ & $\ldots$ \\
\hline J163032.26-243127.9 & SSTc2d J163032.3-243128 & $\ldots$ & $\mathrm{Y}$ & $\ldots$ & $\ldots$ & $\ldots$ & $\ldots$ \\
\hline J163033.26-243038.7 & SSTc2d J163033.2-243039 & $\ldots$ & $\mathrm{Y}$ & $\ldots$ & $\ldots$ & $\ldots$ & $\ldots$ \\
\hline J163033.64-243519.0 & SSTc2d J163033.6-243519 & $\ldots$ & $\mathrm{Y}$ & $\ldots$ & $\ldots$ & $\ldots$ & $\ldots$ \\
\hline J163035.63-243418.9 & 2E 3707 & ROX 39 & $\mathrm{Y}$ & Y & $\mathrm{Y}$ & SFAM 87 & YSO \\
\hline J163036.93-241334.9 & SSTc2d J163036.9-241335 & $\ldots$ & $\mathrm{Y}$ & $\ldots$ & $\mathrm{Y}$ & $\ldots$ & $\ldots$ \\
\hline J163037.85-241206.0 & SFAM 90 & $\ldots$ & $\mathrm{Y}$ & $\ldots$ & $\mathrm{Y}$ & ROS 36 & $\ldots$ \\
\hline J163100.40-241640.4 & SSTc2d J163100.4-241640 & $\ldots$ & $\mathrm{Y}$ & $\ldots$ & $\ldots$ & SFAM 105 & $\ldots$ \\
\hline J163109.63-242554.7 & SSTc2d J163109.6-242555 & $\ldots$ & $\mathrm{Y}$ & $\ldots$ & $\ldots$ & $\ldots$ & $\ldots$ \\
\hline J163109.79-243008.4 & ROC 49 & $\ldots$ & $\ldots$ & $\ldots$ & $\ldots$ & SFAM 108 & $\ldots$ \\
\hline J163115.01-243243.9 & 2MASS J16311501-2432436 & $\ldots$ & $\mathrm{Y}$ & $\mathrm{Y}$ & $\mathrm{Y}$ & $\ldots$ & YSO \\
\hline J163115.75-243402.8 & IRAS 16282-2427 & $\ldots$ & $\mathrm{Y}$ & Y & $\mathrm{Y}$ & $\ldots$ & YSO \\
\hline J163120.14-242928.5 & ROC 52 & $\ldots$ & $\ldots$ & $\ldots$ & $\ldots$ & SFAM 112 & $\ldots$ \\
\hline J163120.18-243001.0 & NTTS $162819-2423 \mathrm{~N}$ & ROXs 43B & $\mathrm{Y}$ & $\mathrm{Y}$ & $\ldots$ & $\ldots$ & YSO \\
\hline J163130.62-243351.6 & SSTc2d J163130.6-243352 & $\ldots$ & $\mathrm{Y}$ & $\ldots$ & $\ldots$ & $\ldots$ & $\ldots$ \\
\hline J163131.09-242719.6 & 6dFGS gJ163131.1-242720 & $\ldots$ & $\mathrm{Y}$ & Y & $\mathrm{Y}$ & ROC 53 & $\mathrm{E}$ \\
\hline J163140.49-245234.7 & SSTc2d J163140.5-245235 & $\ldots$ & $\mathrm{Y}$ & $\ldots$ & $\ldots$ & SFAM 122 & $\ldots$ \\
\hline J163152.10-245615.7 & LDN 1689 IRS 5 & $\ldots$ & $\mathrm{Y}$ & Y & $\mathrm{Y}$ & L1689S1 1 & YSO \\
\hline J163154.49-245217.1 & SSTc2d J163154.5-245217 & $\ldots$ & $\mathrm{Y}$ & $\ldots$ & $\ldots$ & $\ldots$ & $\ldots$ \\
\hline J163159.36-245639.7 & L1689S1 2 & $\ldots$ & $\ldots$ & $\ldots$ & $\ldots$ & SFAM 127 & $\ldots$ \\
\hline J163159.51-252918.7 & SSTc2d J163159.5-252919 & $\ldots$ & $\mathrm{Y}$ & $\ldots$ & $\ldots$ & $\ldots$ & $\ldots$ \\
\hline J163200.97-245643.3 & WLY $2-67$ & $\ldots$ & $\mathrm{Y}$ & $\mathrm{Y}$ & $\mathrm{Y}$ & L1689S1 3 & YSO \\
\hline J163208.05-253016.3 & $\ldots$ & $\ldots$ & $\ldots$ & $\ldots$ & $\ldots$ & SFAM 130 & $\ldots$ \\
\hline J163210.77-243827.6 & SSTc2d J163210.8-243827 & $\ldots$ & $\mathrm{Y}$ & $\ldots$ & $\mathrm{Y}$ & $\ldots$ & $\ldots$ \\
\hline J163211.08-243651.1 & SSTc2d J163211.1-243652 & $\ldots$ & $\mathrm{Y}$ & $\ldots$ & $\ldots$ & $\ldots$ & $\ldots$ \\
\hline J163211.79-244021.8 & V2248 Oph & 1RXS J163212.8-244013 & $\mathrm{Y}$ & $\mathrm{Y}$ & $\mathrm{Y}$ & $\ldots$ & YSO \\
\hline J163214.16-252344.5 & SSTc2d J163214.1-252345 & $\ldots$ & $\mathrm{Y}$ & $\ldots$ & $\ldots$ & NVSS 163214-252344 & $\ldots$ \\
\hline J163227.41-243951.4 & SSTc2d J163227.4-243951 & $\ldots$ & $\mathrm{Y}$ & $\ldots$ & $\ldots$ & $\ldots$ & $\ldots$ \\
\hline J163231.17-244014.6 & SSTc2d J163231.2-244014 & $\ldots$ & $\mathrm{Y}$ & $\ldots$ & $\ldots$ & $\ldots$ & $\ldots$ \\
\hline J163245.23-243647.4 & SFAM 200 & $\ldots$ & $\mathrm{Y}$ & $\ldots$ & $\ldots$ & $\ldots$ & $\ldots$ \\
\hline J163421.10-235625.1 & WSB 80 & RX J1634.3-2356 & $\ldots$ & $\mathrm{Y}$ & $\mathrm{Y}$ & $\ldots$ & YSO \\
\hline J163436.01-235614.5 & $\ldots$ & $\ldots$ & $\ldots$ & $\ldots$ & $\ldots$ & NVSS 163436-235611 & $\ldots$ \\
\hline J163551.89-242253.6 & SSTc2d J163551.9-242253 & $\ldots$ & $\mathrm{Y}$ & $\ldots$ & $\ldots$ & $\ldots$ & $\ldots$ \\
\hline
\end{tabular}


Table 3

(Continued)

\begin{tabular}{|c|c|c|c|c|c|c|c|}
\hline \multirow[t]{2}{*}{ GB-VLA Name } & \multirow[t]{2}{*}{ Other Names } & \multirow[b]{2}{*}{ X-Ray ${ }^{\mathrm{a}}$} & \multicolumn{3}{|c|}{ Infrared $^{\mathrm{b}, \mathrm{c}}$} & \multirow[t]{2}{*}{ Radio $^{\mathrm{d}}$} & \multirow[t]{2}{*}{ Object Type } \\
\hline & & & $S S T$ & $2 \mathrm{M}$ & WISE & & \\
\hline J163557.74-241447.9 & $\ldots$ & $\ldots$ & $\ldots$ & $\ldots$ & $\ldots$ & NVSS 163557-241446 & $\ldots$ \\
\hline J163615.79-242159.8 & SSTc2d J163615.8-242160 & $\ldots$ & $\mathrm{Y}$ & $\ldots$ & $\ldots$ & $\ldots$ & $\ldots$ \\
\hline J163617.50-242555.4 & SSTc2d J163617.5-242555 & $\ldots$ & $\mathrm{Y}$ & $\ldots$ & $\ldots$ & SFAM 212 & $\ldots$ \\
\hline J163639.40-241710.3 & SSTc2d J163639.4-241710 & $\ldots$ & $\mathrm{Y}$ & $\ldots$ & $\mathrm{Y}$ & $\ldots$ & $\ldots$ \\
\hline J163949.54-235939.0 & SSTc2d J163949.5-235939 & $\ldots$ & $\mathrm{Y}$ & $\ldots$ & $\mathrm{Y}$ & $\ldots$ & $\ldots$ \\
\hline
\end{tabular}

Notes.

a ROXRA: Grosso et al. 2000; A and BF: Imanishi et al. 2003; DROXO: Pillitteri et al. 2010; ROX: Montmerle et al. 1983; ROXs: Bouvier \& Appenzeller 1992; 1RXS: Voges et al. 1999; and RX: Martin et al. 1998.

b SST: Evans et al. 2009; 2M: Cutri et al. 2003; and WISE: Cutri et al. 2012.

${ }^{c}$ For GBDS VLA J162722.96-242236.6, 2MASS, and SST data from Marsh et al. (2010).

d ROC and ROS: André et al. 1987; GDS: Gagné et al. 2004; NVSS: Condon et al. 1998; LFAM: Leous et al. 1991 ; SFAM: Stine et al. 1988.

Table 4

Young Stellar Object Candidates Based Just in Their Radio Properties

\begin{tabular}{|c|c|c|c|}
\hline \multirow[t]{2}{*}{ GB-VLA Name } & \multicolumn{2}{|c|}{ Flux Properties } & \multirow[t]{2}{*}{ Spectral Index } \\
\hline & $\operatorname{Var}_{4.5}(\%)$ & $\operatorname{Var}_{.7 .5}(\%)$ & \\
\hline J162605.29-243436.6 & & $33.9 \pm 27.2$ & $>1.34 \pm 0.14$ \\
\hline J162632.78-241627.5 & $38.8 \pm 18.7$ & $32.1 \pm 18.4$ & $0.86 \pm 0.49$ \\
\hline J162633.16-245246.7 & $30.7 \pm 5.5$ & $49.3 \pm 5.3$ & $0.27 \pm 0.18$ \\
\hline J162637.27-244553.9 & $52.8 \pm 8.4$ & $>78.0 \pm 3.0$ & $-0.96 \pm 0.40$ \\
\hline J162641.11-245855.8 & $67.8 \pm 3.3$ & $81.0 \pm 2.3$ & $0.5 \pm 0.18$ \\
\hline J162646.36-242002.0 & $15.3 \pm 6.4$ & $21.6 \pm 5.7$ & $0.66 \pm 0.17$ \\
\hline J162647.23-243620.3 & $21.5 \pm 6.9$ & $22.0 \pm 7.9$ & $0.31 \pm 0.19$ \\
\hline J162702.11-243842.5 & $>55.5 \pm 4.3$ & $68.6 \pm 7.3$ & $0.04 \pm 0.26$ \\
\hline J162752.30-242929.9 & $>43.8 \pm 14.8$ & $>17.1 \pm 16.9$ & $0.38 \pm 0.48$ \\
\hline J162756.01-244810.7 & $\ldots$ & $>55.8 \pm 15.9$ & $>1.53 \pm 0.14$ \\
\hline J162803.51-242131.2 & $>43.2 \pm 5.5$ & $\ldots$ & $<0.08 \pm 0.14$ \\
\hline J163032.26-243127.9 & $42.5 \pm 5.3$ & $43.5 \pm 6.2$ & $0.32 \pm 0.20$ \\
\hline J163033.26-243038.7 & $>60.7 \pm 5.9$ & $>24.1 \pm 12.1$ & $0.53 \pm 0.78$ \\
\hline J163033.64-243519.0 & $>10.6 \pm 24.5$ & $38.1 \pm 19.6$ & $0.27 \pm 0.39$ \\
\hline J163058.02-243441.3 & $29.4 \pm 12.7$ & $>26.3 \pm 8.7$ & $0.2 \pm 0.43$ \\
\hline J163151.93-245617.4 & $93.3 \pm 0.7$ & $97.5 \pm 0.4$ & $0.88 \pm 0.22$ \\
\hline J163437.30-235946.2 & $17.5 \pm 8.2$ & $>33.1 \pm 8.4$ & $0.46 \pm 0.24$ \\
\hline J163626.93-242117.8 & $29.8 \pm 15.7$ & $>35.9 \pm 10.4$ & $0.26 \pm 0.41$ \\
\hline
\end{tabular}

\section{DISCUSSION}

\subsection{Background Sources}

It is clear from Section 3 that a large fraction of the radio sources detected here are background objects. To examine their statistics, we will concentrate on the core region (for which we have a continuous coverage at uniform sensitivity) and on the observations at $4.5 \mathrm{GHz}$, which are more appropriate than those at $7.5 \mathrm{GHz}$ for extragalactic objects, since those usually have negative spectral indices. Fomalont et al. (1991) showed that the source counts at $5 \mathrm{GHz}$ are appropriately described by

$$
\left(\frac{N}{\operatorname{arcmin}^{2}}\right)=0.42 \pm 0.05\left(\frac{S}{30 \mu \mathrm{Jy}}\right)^{-1.18 \pm 0.19}
$$

According to those counts, the number of sources brighter than $150 \mu \mathrm{Jy}$ (the minimum flux of the sources that we detected; see Table 1) expected in the $1185 \mathrm{arcmin}^{2}$ covered by our Ophiuchus core observations is $75 \pm 9$. The number of objects classified as extragalactic in the core is 3 , and there are 79 unclassified sources in that region. As we mentioned earlier, most of these unclassified source are likely extragalactic, with only a small

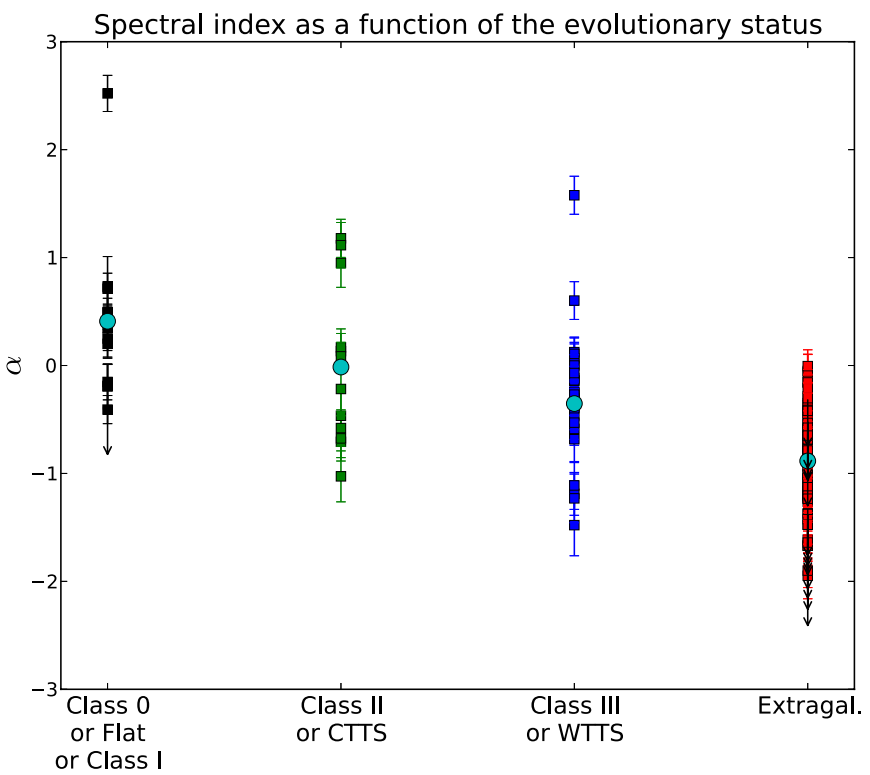

Figure 3. Spectral index as a function of the YSO evolutionary status. Extragalactic objects are also displayed for reference. The individual sources are shown with their error bars, and the turquoise circles indicate the mean spectral index for each category.

(A color version of this figure is available in the online journal.)

contribution (of at most 15\%) of YSOs. This means that about 67 of the unclassified sources in the core are extragalactic, and that the total number of observed extragalactic sources in that region is about 70, in excellent agreement with the count predicted by Fomalont et al. (1991). Note that the counts would in fact also be consistent with the possibility that all the unidentified sources are extragalactic.

\subsection{Radio Properties of the YSO Population}

In Section 3, we mentioned that 56 of the radio sources detected here are associated with young stars. The spectral type and evolutionary status for most of these objects are known (see Table 5) and can be compared to their radio properties. In Figure 3, the radio spectral index is plotted as a function of evolutionary status. There is a clear tendency for more evolved YSOs to have a smaller (i.e., more negative) spectral index. The younger (Class 0, flat spectrum, and Class I) sources have a mean spectral index of order 0.5 , indicating that the 
Table 5

Young Stellar Objects Detected in the Radio Observations

\begin{tabular}{|c|c|c|c|c|c|c|c|}
\hline GB-VLA Name & $\begin{array}{c}\text { Spectral } \\
\text { Type }\end{array}$ & $\begin{array}{c}\text { SED } \\
\text { Clasification }\end{array}$ & Var. $^{a}$ & Pol. $^{\mathrm{a}}$ & $\alpha^{\mathrm{a}}$ & X-Ray & Ref. $^{b}$ \\
\hline $\mathrm{J} 162550.51-243914.7$ & K5.5 & Class III & $\mathrm{Y}$ & $\mathrm{N}$ & $\mathrm{N}$ & $\mathrm{Y}$ & 1 \\
\hline $\mathrm{J} 162556.09-243015.3$ & M5 & Class III & $\mathrm{N}$ & $\mathrm{N}$ & $\mathrm{F}$ & $\mathrm{N}$ & 1 \\
\hline $\mathrm{J} 162557.51-243032.1$ & K6 & Class III & $\mathrm{Y}$ & $\mathrm{Y}$ & $\mathrm{N}$ & $\mathrm{Y}$ & 1 \\
\hline $\mathrm{J} 162603.01-242336.4$ & K0 & Class III & $\mathrm{Y}$ & $\mathrm{Y}$ & $\mathrm{F}$ & $\mathrm{Y}$ & 1,2 \\
\hline $\mathrm{J} 162607.63-242741.7$ & - & Class III & $\mathrm{Y}$ & $\mathrm{N}$ & $\mathrm{N}$ & $\mathrm{Y}$ & 1 \\
\hline $\mathrm{J} 162610.32-242054.9$ & M0 & Class II & $\mathrm{Y}$ & - & $\mathrm{P}$ & $\mathrm{Y}$ & 1 \\
\hline J162616.84-242223.5 & K6 & Class II & $\mathrm{Y}$ & $\mathrm{N}$ & $\mathrm{F}$ & $\mathrm{Y}$ & 1 \\
\hline $\mathrm{J} 162621.72-242250.7$ & - & Class I & $\mathrm{N}$ & - & $\mathrm{P}$ & $\mathrm{N}$ & 1 \\
\hline $\mathrm{J} 162622.38-242253.3$ & K8 & Class II & $\mathrm{Y}$ & $\mathrm{N}$ & $\mathrm{N}$ & $\mathrm{Y}$ & 1 \\
\hline J162623.57-242439.6 & M0 & FS & $\mathrm{N}$ & - & $\mathrm{F}$ & $\mathrm{Y}$ & 1 \\
\hline $\mathrm{J} 162624.08-241613.5$ & K5.5 & Class II & $\mathrm{N}$ & - & $\mathrm{P}$ & $\mathrm{Y}$ & 1,2 \\
\hline $\mathrm{J} 162625.62-242429.2$ & - & Class I & $\mathrm{N}$ & - & $\mathrm{F}$ & $\mathrm{N}$ & 3 \\
\hline $\mathrm{J} 162626.31-242430.3^{\mathrm{c}}$ & - & Class 0 (?) & $\mathrm{Y}$ & - & $\mathrm{P}$ & $\mathrm{N}$ & 3 \\
\hline J162626.39-242430.9 & - & Class 0 & $\mathrm{~N}$ & - & $\mathrm{P}$ & $\mathrm{N}$ & 4 \\
\hline J162629.67-241905.8 & M1.5 & Class III & $\mathrm{Y}$ & $\mathrm{N}$ & $\mathrm{N}$ & $\mathrm{Y}$ & 1 \\
\hline $\mathrm{J} 162634.17-242328.4$ & B4 & Class III & $\mathrm{N}$ & $\mathrm{Y}$ & $\mathrm{N}$ & $\mathrm{Y}$ & 1 \\
\hline $\mathrm{J} 162642.44-242626.1$ & - & Class III & $\mathrm{Y}$ & $\mathrm{N}$ & $\mathrm{F}$ & $\mathrm{Y}$ & 1 \\
\hline J162643.76-241633.4 & M0 & Class III & $\mathrm{N}$ & - & $\mathrm{F}$ & $\mathrm{Y}$ & 1,2 \\
\hline J162649.23-242003.3 & K6 & Class III & $\mathrm{Y}$ & - & $\mathrm{N}$ & $\mathrm{Y}$ & 1 \\
\hline $\mathrm{J} 162658.38-242130.5$ & - & Class II & $\mathrm{Y}$ & - & $\mathrm{N}$ & $\mathrm{Y}$ & 1 \\
\hline J162659.16-243458.9 & - & Class I & $\mathrm{N}$ & $\mathrm{N}$ & $\mathrm{P}$ & $\mathrm{Y}$ & 1 \\
\hline $\mathrm{J} 162702.33-243727.3$ & B8-A7 & Class I & $\mathrm{N}$ & - & $\mathrm{P}$ & $\mathrm{Y}$ & 1 \\
\hline $\mathrm{J} 162705.16-242007.8$ & - & Class III & $\mathrm{N}$ & - & $\mathrm{N}$ & $\mathrm{Y}$ & 1 \\
\hline $\mathrm{J} 162705.25-243629.8$ & - & Class I & $\mathrm{N}$ & - & $\mathrm{F}$ & $\mathrm{N}$ & 1 \\
\hline $\mathrm{J} 162705.96-242618.9$ & - & Class II & $\mathrm{Y}$ & - & $\mathrm{N}$ & $\mathrm{Y}$ & 1 \\
\hline J162709.41-243719.0 & - & Class I & $\mathrm{N}$ & $\mathrm{N}$ & $\mathrm{P}$ & $\mathrm{Y}$ & 1 \\
\hline J162715.69-243845.7 & $\mathrm{BD}$ ? & - & $\mathrm{N}$ & $\mathrm{N}$ & $\mathrm{F}$ & $\mathrm{N}$ & 5 \\
\hline J162718.17-242852.9 & F7 & Class III & $\mathrm{Y}$ & - & $\mathrm{N}$ & $\mathrm{Y}$ & 1 \\
\hline $\mathrm{J} 162719.50-244140.4$ & M2.5 & Class III & $\mathrm{N}$ & - & $\mathrm{N}$ & $\mathrm{Y}$ & 1 \\
\hline $\mathrm{J} 162721.81-244336.0$ & M3 & Class III & $\mathrm{Y}$ & $\mathrm{N}$ & $\mathrm{F}$ & $\mathrm{Y}$ & 1 \\
\hline $\mathrm{J} 162721.97-242940.0$ & - & Class II & $\mathrm{N}$ & - & $\mathrm{N}$ & $\mathrm{Y}$ & 1 \\
\hline J162722.96-242236.6 & $\mathrm{BD}$ & - & $\mathrm{Y}$ & - & $\mathrm{N}$ & $\mathrm{N}$ & 6 \\
\hline J162724.19-242929.6 & - & Class III & $\mathrm{Y}$ & - & $\mathrm{N}$ & $\mathrm{Y}$ & 1 \\
\hline $\mathrm{J} 162726.90-244050.8$ & - & Class I & $\mathrm{N}$ & - & $\mathrm{N}$ & $\mathrm{Y}$ & 1 \\
\hline $\mathrm{J} 162727.36-243116.8$ & M0 & Class II & $\mathrm{Y}$ & $\mathrm{N}$ & $\mathrm{N}$ & $\mathrm{Y}$ & 1 \\
\hline $\mathrm{J} 162728.00-243933.7$ & K8 & Class I & $\mathrm{N}$ & $\mathrm{N}$ & $\mathrm{F}$ & $\mathrm{Y}$ & 1 \\
\hline $\mathrm{J} 162730.82-244727.2$ & M1 & Class III & $\mathrm{Y}$ & - & $\mathrm{N}$ & $\mathrm{Y}$ & 5,7 \\
\hline J162731.05-243403.4 & - & Class III & $\mathrm{Y}$ & $\mathrm{N}$ & $\mathrm{P}$ & $\mathrm{Y}$ & 1 \\
\hline $\mathrm{J} 162732.68-243324.5$ & M2 & Class II & $\mathrm{Y}$ & $\mathrm{N}$ & $\mathrm{F}$ & $\mathrm{Y}$ & 8 \\
\hline J162739.41-243915.8 & K5 & Class II & $\mathrm{N}$ & - & $\mathrm{P}$ & $\mathrm{Y}$ & 1 \\
\hline J162741.49-243537.6 & - & Class III & $\mathrm{Y}$ & - & $\mathrm{N}$ & $\mathrm{Y}$ & 1 \\
\hline $\mathrm{J} 162749.85-242540.5$ & A7 & Class III & $\mathrm{N}$ & $\mathrm{Y}$ & $\mathrm{F}$ & $\mathrm{N}$ & 1 \\
\hline $\mathrm{J} 162751.80-243145.9$ & - & Class I & $\mathrm{N}$ & $\mathrm{N}$ & $\mathrm{N}$ & $\mathrm{Y}$ & 7 \\
\hline J162751.89-244630.1 & - & Class III & $\mathrm{Y}$ & $\mathrm{N}$ & $\mathrm{N}$ & $\mathrm{Y}$ & 7 \\
\hline $\mathrm{J} 162752.08-244050.5$ & K7 & - & $\mathrm{Y}$ & $\mathrm{Y}$ & $\mathrm{P}$ & $\mathrm{Y}$ & 9 \\
\hline J162757.81-244001.9 & K7 & Class III & $\mathrm{Y}$ & - & $\mathrm{N}$ & $\mathrm{Y}$ & 1,10 \\
\hline $\mathrm{J} 162759.95-244819.5$ & M4.75 & WTTS & $\mathrm{Y}$ & - & $\mathrm{P}$ & $\mathrm{Y}$ & 1,11 \\
\hline $\mathrm{J} 162804.65-243456.6$ & - & Class II & $\mathrm{Y}$ & - & $\mathrm{N}$ & $\mathrm{Y}$ & 8 \\
\hline J163035.63-243418.9 & K5 & CTTS & $\mathrm{N}$ & $\mathrm{N}$ & $\mathrm{F}$ & $\mathrm{Y}$ & 9,12 \\
\hline J163115.01-243243.9 & M0 & WTTS & $\mathrm{Y}$ & Y & $\mathrm{N}$ & $\mathrm{N}$ & 9,13 \\
\hline $\mathrm{J} 163115.75-243402.8$ & K6 & WTTS & $\mathrm{Y}$ & $\mathrm{N}$ & $\mathrm{F}$ & $\mathrm{N}$ & 9,12 \\
\hline J163120.18-243001.0 & $\mathrm{K} 3 \mathrm{e}$ & WTTS & $\mathrm{Y}$ & $\mathrm{N}$ & $\mathrm{F}$ & $\mathrm{Y}$ & 9,14 \\
\hline $\mathrm{J} 163152.10-245615.7$ & M3 & FS & $\mathrm{Y}$ & - & $\mathrm{P}$ & $\mathrm{N}$ & 8 \\
\hline J163200.97-245643.3 & K5 & Class I & $\mathrm{Y}$ & $\mathrm{Y}$ & $\mathrm{P}$ & $\mathrm{N}$ & 8 \\
\hline $\mathrm{J} 163211.79-244021.8$ & M3 & WTTS/Class II & $\mathrm{N}$ & $\mathrm{Y}$ & $\mathrm{N}$ & $\mathrm{Y}$ & 8 \\
\hline $\mathrm{J} 163421.10-235625.1$ & M4.5 & WTTS & $\mathrm{Y}$ & - & $\mathrm{N}$ & $\mathrm{Y}$ & 11,9 \\
\hline
\end{tabular}

\section{Notes.}

${ }^{\text {a }}$ Var. $=\mathrm{Y}$ when the source variability is higher than $50 \%$ in at least one frequency; $\mathrm{N}$ when it is lower. Pol. $=\mathrm{Y}$ when circular polarization is detected and $\mathrm{N}$ when it is not. Sources in the outer fields for which the polarization could not be assessed are shown as "-." $\alpha$ refers to the spectral index, and is given as $\mathrm{P}$ (for positive) when it is higher than 0.2 ; $\mathrm{F}$ (for flat) when it is between -0.2 and +0.2 , and $\mathrm{N}$ (for negative) when it is lower than -0.2 . X-ray $=\mathrm{Y}$ when there is an $\mathrm{X}$-ray flux reported in literature, $\mathrm{N}$ when it is not. b 1, Wilking et al. 2005; 2, Gagné et al. 2004; 3, Murillo \& Lai 2013; 4, Andre et al. 1993; 5, Alves de Oliveira et al. 2010; 6, Marsh et al. 2010; 7, Pillitteri et al. 2010; 8, McClure et al. 2010; 9, Cieza et al. 2007; 10, Wilking et al. 2001; 11, Martin et al. 1998; 12, Wahhaj et al. 2010; 13, Bouvier \& Appenzeller 1992; 14, Torres et al. 2006.

c This source corresponds to VLA1623B; as discussed in the text, it may be a young star or an outflow knot feature. dominant emission process is somewhat optically thick free-free emission. The older (Class II and III) sources, on the other hand, have a slightly negative mean spectral index, suggestive either of optically thin free-free emission or of gyrosynchrotron radiation (see Section 1). In particular, there is a significant population of Class II/III objects with $\alpha<-0.1$, and which are most likely non-thermal emitters. Note, however, that the boundaries are not sharp since some very young objects have slightly negative indices, while one of the more evolved YSOs reach a spectral index above one. As expected, the extragalactic objects in our sample typically have negative spectral indices, with a mean value of order -1 .

The radio variability is shown as a function of evolutionary status in Figure 4. It is clear that younger sources are, on average, less variable than their more evolved counterparts. Since non-thermal emitters are often strongly variable, the measured increase in variability confirms the conclusion drawn above from the spectral index analysis that there is a significant population of non-thermal sources among the Class II/III sources in our sample. A final piece of information supports that conclusion: The radio flux appears to also be, on average, higher for more evolved sources (Figure 5), particularly from objects of Class III as compared to those of Class II. Since younger sources experience more intense outflow activity, they will be stronger thermal emitters than more evolved objects (this is consistent with the observed variability and spectral index trends described above). The stronger average radio flux for more evolved sources must, therefore, indicate that a different mechanism dominates the radio emission as young stars age. The most natural candidate is, again, gyrosynchrotron emission. It is important to point out, however, that young YSOs might well intrinsically emit as much non-thermal radiation as their older siblings, but because of their dense ionized winds, such emission might be absorbed by the optically thick free-free emission (e.g., Forbrich et al. 2007; Deller et al. 2013; see also Section 5.3).

The previous discussion shows that there exists a significant population of non-thermal radio sources in our sample of detected YSOs. From Table 5, we can see that 25 of the 55 young stars in our sample (45\%) either are polarized, or have high variability and a negative spectral index. These source are almost certainly non-thermal and, as expected, they are mostly somewhat evolved $-64 \%$ of them are Class III and/or WTTS. There might be an even larger population of non-thermal YSOs in our sample since those sources which are either highly variable but with positive spectral index, or steady but with a negative spectral index might also fall in that category. Finally, if any of the unclassified sources given in Table 4 are indeed young stellar sources, they must also be non-thermal.

A final trend must be mentioned here. Out of the 36 detected YSOs with known spectral type, 31 fall in the range K0 to M5 (Figure 6), while no G-type star, and only one F, two A, and one B stars are detected. This points to a smaller number of radio-bright early-type stars compared to what would be expected on the basis of a typical stellar IMF alone. For instance, according to the Kroupa (2001) IMF, roughly $50 \%$ of all stars have a spectral type between K0 and M5. Could this be due to an IMF inherently deficient in higher-mass stars? Hsu et al. (2013) find that the pre-main-sequence population of the L1641 dark cloud in Orion has a deficit of higher-mass stars when compared to a compact region like the Orion Nebula Cluster, and suggest that the environment may play a role in determining the high-mass end of the IMF. We note, however, that Erickson et al. 
Variability as a function of evolutionary status
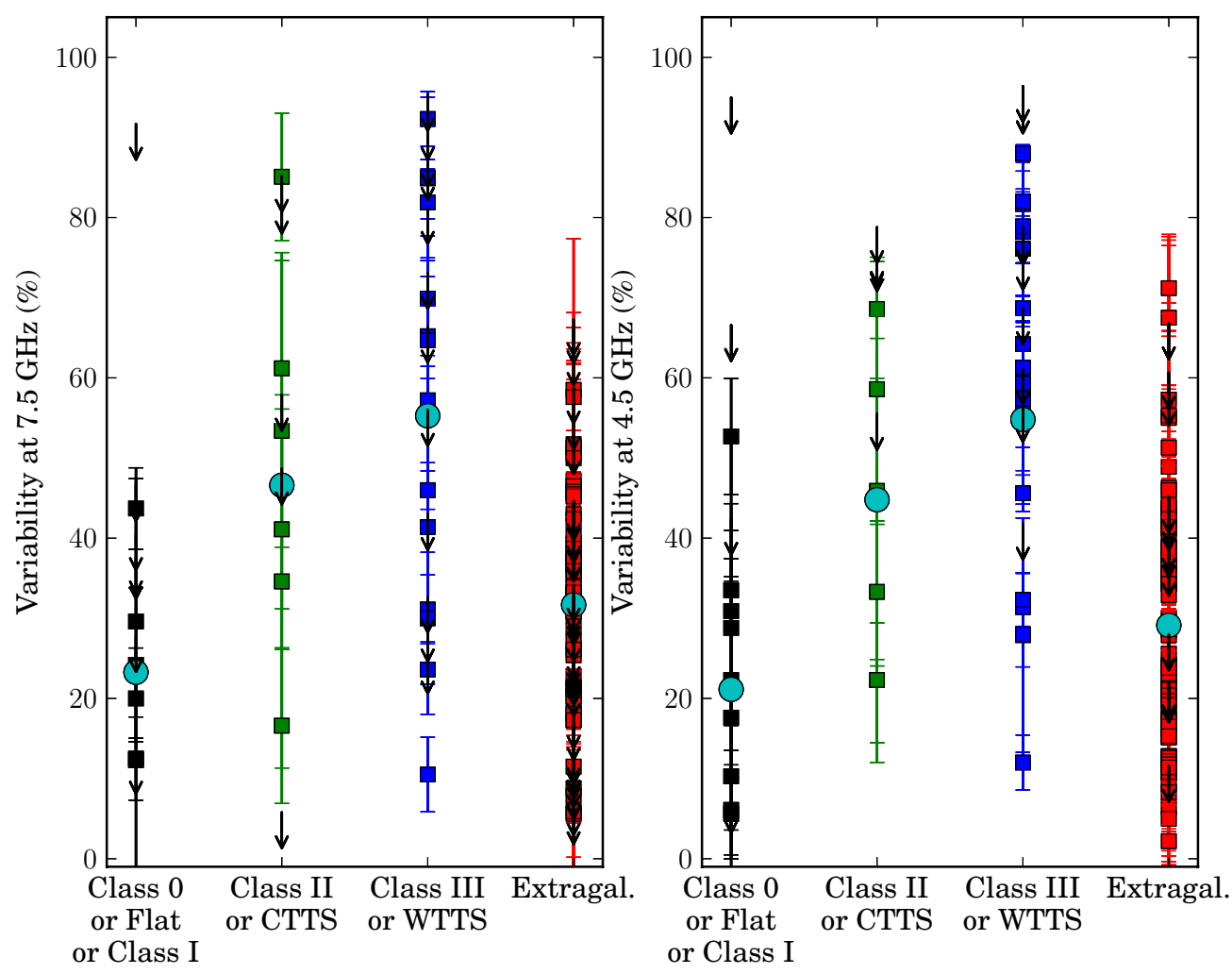

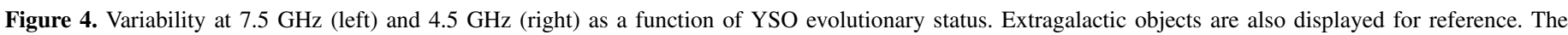
individual sources are shown with their error bars, and the turquoise circles indicate the mean variability for each category.

(A color version of this figure is available in the online journal.)

Flux density as a function of evolutionary status
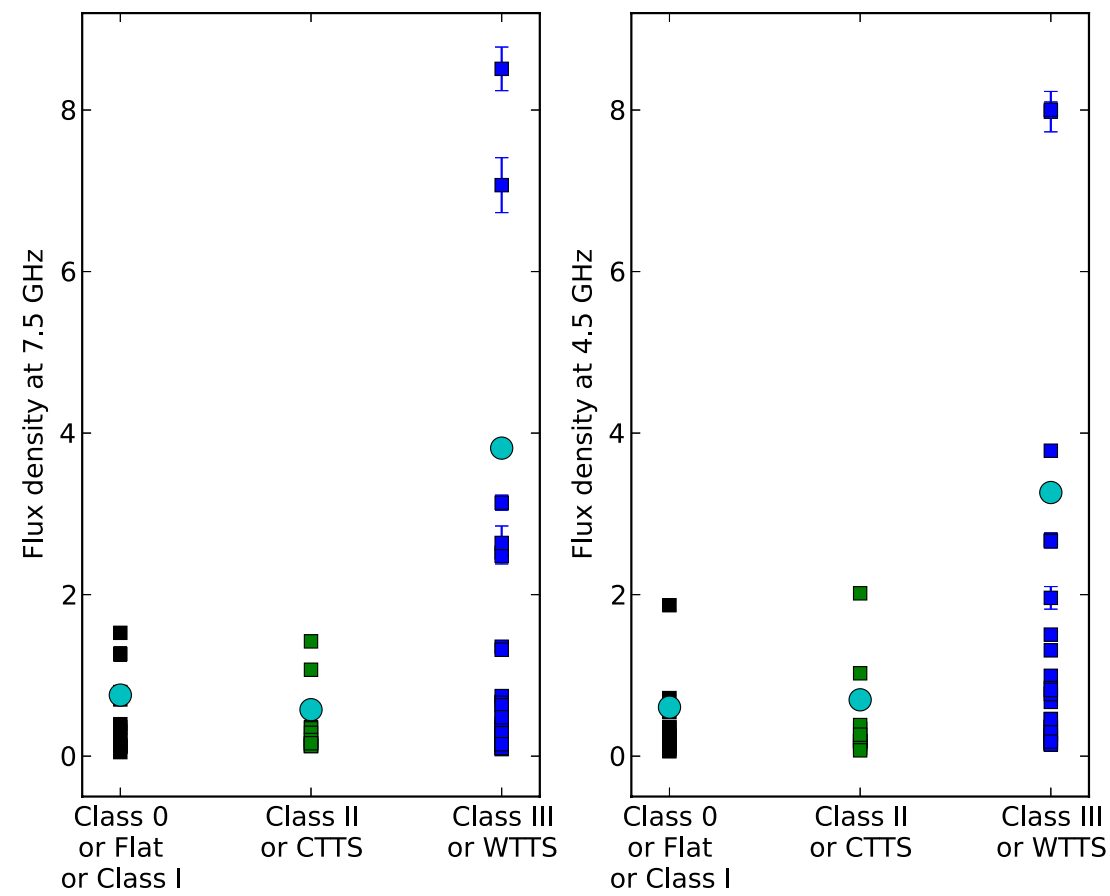

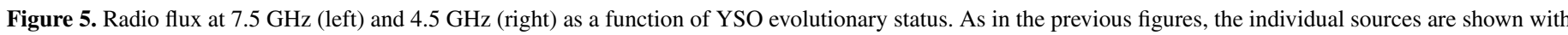
their error bars, and the black circles indicate the mean flux for each category.

(A color version of this figure is available in the online journal.) 
Flux density as a function of the spectral type
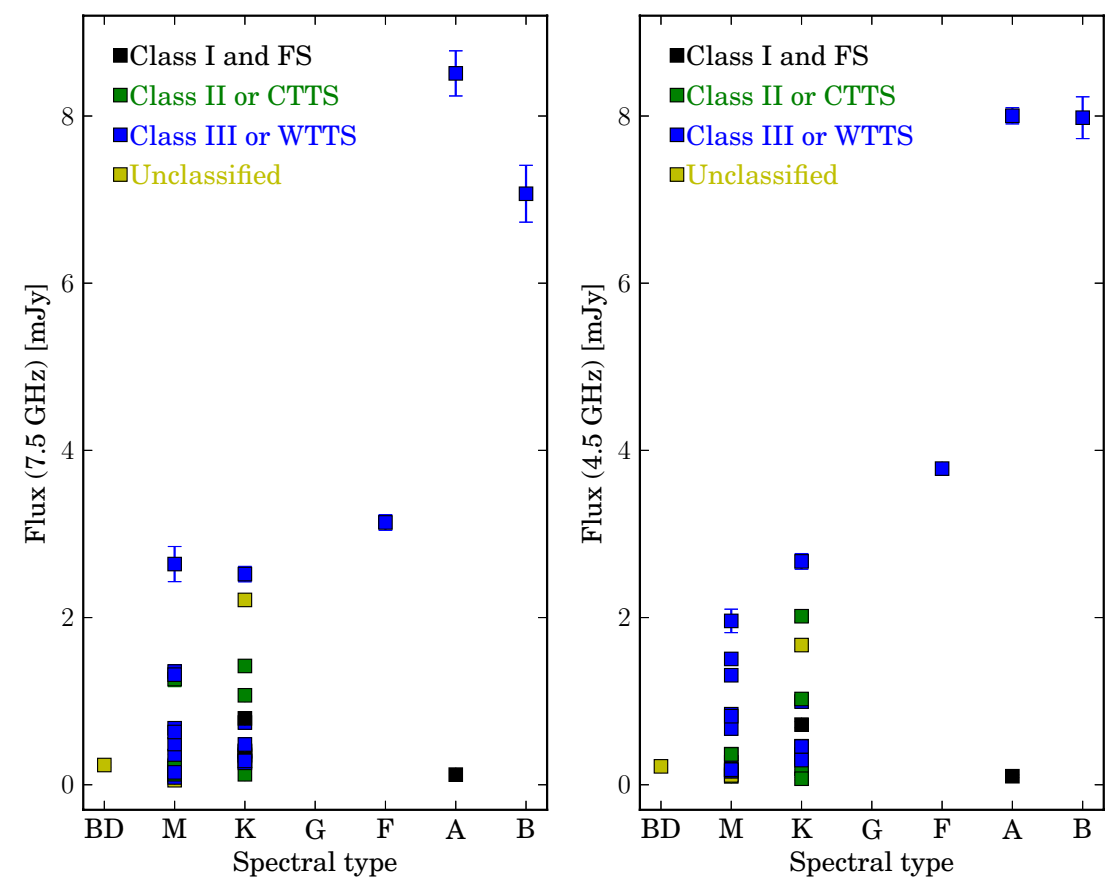

Figure 6. Radio flux at $7.5 \mathrm{GHz}$ (left) and $4.5 \mathrm{GHz}$ (right) as a function of YSO spectral type. Colors indicate the evolutionary of the object as explained at the top left of the diagrams.

(A color version of this figure is available in the online journal.)

(2011) constructed an IMF for an extinction-limited sample of 123 YSOs in L1688 and found it to be consistent with that of field star for $M>0.2 M_{\odot}$. Alternatively, our finding might indicate that the fraction of radio-bright young stars increases for later type stars, in agreement with the trend noticed for more evolved stars by Berger et al. (2010). On the other hand, the detected $\mathrm{F} / \mathrm{A} / \mathrm{B}$ stars are on average significantly brighter at radio wavelengths than their $\mathrm{M}$ and $\mathrm{K}$ counterparts, confirming that (for earlier type stars), there is a good correlation between the bolometric and radio luminosities.

It is also possible that the lower fraction of radio-bright early-type stars could be the result of an observational bias. The problem is that it is very difficult to identify YSOs with spectral types G, F, A, and B, unless they are actively accreting, as inferred from certain lines strongly in emission, like $\mathrm{H} \alpha$ $\lambda 6563$ and $\mathrm{He}_{\mathrm{I}} \lambda \lambda 5875,6676$, and forbidden lines like [O I] or [S II], or have infrared excess emission originating in a circumstellar disk. While for T Tauri stars we have well-defined observables to identify the full YSO population, for the earliertype counterparts this is not the case. A strong Li I $\lambda 6707$ absorption line, in excess of what is observed in young mainsequence stars like the Pleiades (Briceño et al. 1997, 1998), does not imply youth in $\mathrm{G}$ and earlier-type stars because they do not deplete Li I during their pre-main-sequence phase. The strength of the $\mathrm{NaI}(\lambda \lambda 8183,8195)$ doublet, which allows to distinguish low surface gravity stars still contracting toward the main sequence (e.g., Martin et al. 1996; Slesnick et al. 2006; Downes et al. 2008; Lodieu et al. 2011; Schlieder et al. 2012), breaks down for spectral types earlier than about M0. Strong X-ray emission, characteristic of late F- and G- through M-type young stars, unless combined with other criteria, can suffer from significant contamination by young main-sequence stars with ages up to $\sim 100 \mathrm{Myr}$ (Briceño et al. 1997). So far, the best way to determine the full membership of G- through B-type YSOs seems to be selecting as members objects which satisfy as primary criteria radial velocities and proper motions (if possible, e.g., Hsu et al. 2013), combine these with other characteristics like X-ray emission, emission lines, infrared excesses, and place them in an H-R diagram, which requires a reasonable knowledge of the distance to each and every object, or assumption of a common distance to a group or cluster. If, as suggested by the trend observed in Figure 5, Class III sources are indeed, on average, brighter radio sources than Class II YSOs, the fact that the early-type sample may be biased toward the later could be affecting our result.

\subsection{The Radio-X-Ray Relation}

Güdel \& Benz (1993) and Benz \& Güdel (1994) showed that the radio and X-ray emissions of magnetically active stars are correlated by a relation of the form

$$
\frac{L_{X}}{L_{R}}=\kappa \cdot 10^{15.5 \pm 1} \quad[\mathrm{~Hz}],
$$

where $\kappa$ is unity for dMe and dK stars, BY Dra-type binaries, and RS CVn binaries with two subgiants. For classical RS CVn binaries, Algol systems, FK Com stars, and Post T Tauri stars $\kappa \approx 0.17$. The radio observations used to establish this relation were obtained mainly at $4.8 \mathrm{GHz}$ and, in some cases, at $8.4 \mathrm{GHz}$.

Of the 55 young stars in our sample, 35 have known X-ray counterparts, and can be used to study the $L_{X} / L_{R}$ relation for YSOs in a statistically significant fashion. Note that we corrected all X-ray luminosities to the distance of $120 \mathrm{pc}$ adopted in this work. In Figure 7, we show the X-ray luminosities of the YSOs as a function of their radio luminosities at both radio frequencies observed in this work. In agreement with the results obtained by Gagné et al. (2004) and Forbrich et al. (2010), $L_{X} / L_{R} \leqslant 10^{15.5}$ for our sample. Indeed, a relation $L_{X} / L_{R} \approx 10^{14 \pm 1}$ provides a good match to the distribution of points in this plot. This is equivalent, in terms of the Güdel-Benz relation, to $\kappa=0.03$ for 


\section{Güdel-Benz relation for YSOs in $\rho$-Ophiuchus}
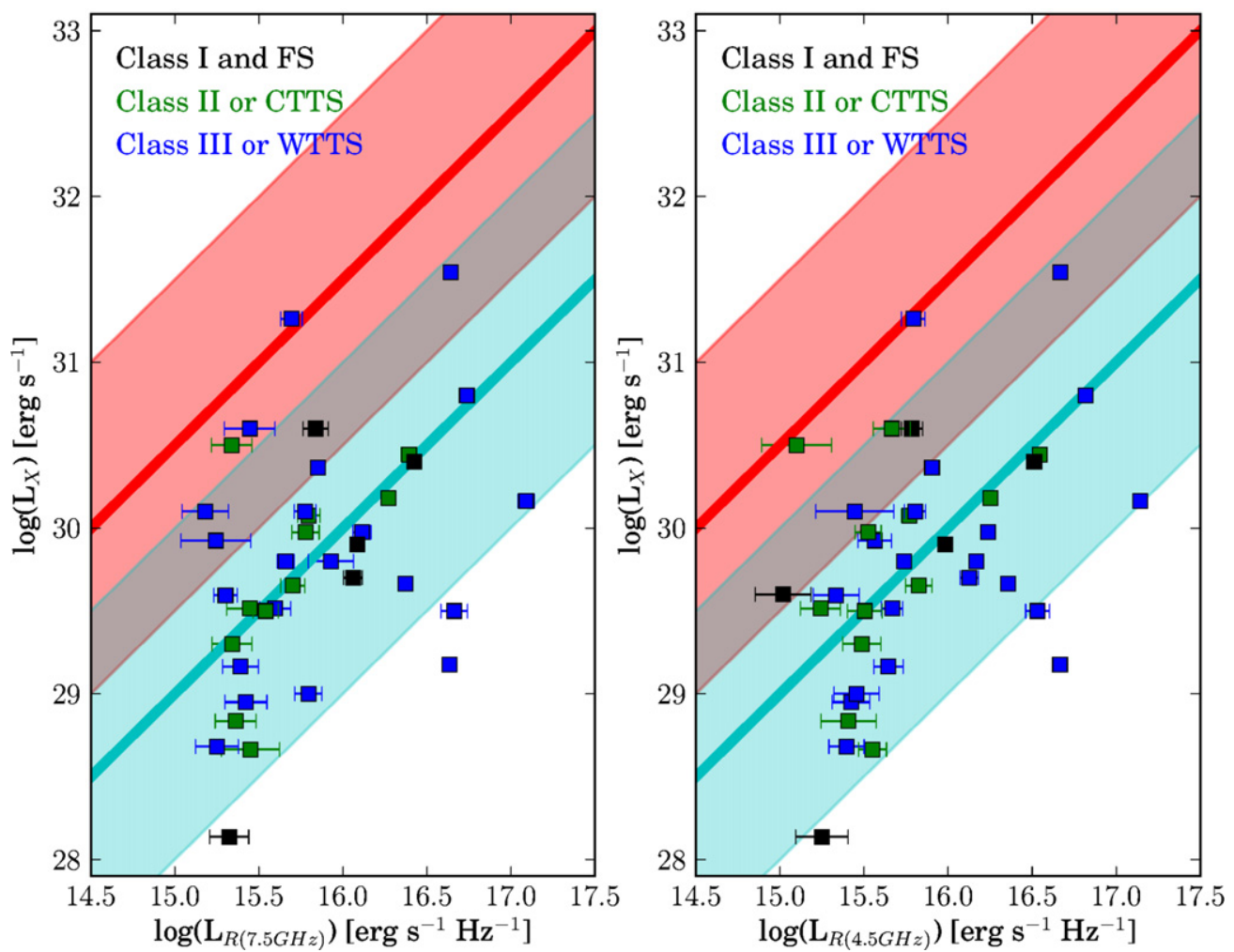

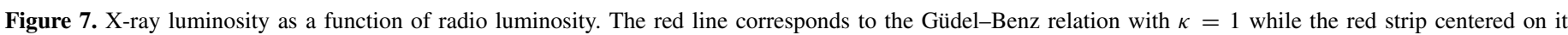

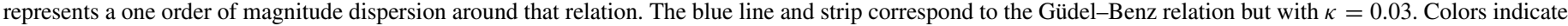
the evolutionary status of the object as explained at the top left of the diagram.

(A color version of this figure is available in the online journal.)

YSOs. We argue that this results provides an extension of the relation valid for YSOs.

\section{COMMENTS ON SOME INDIVIDUAL SOURCES}

\subsection{VLA 1623}

A total of four radio sources associated with the Class 0 source VLA 1623 have been reported in the literature. Bontemps \& Andre (1997; see their Figures 1 and 2) reported on the detection of three roughly aligned objects that they called A, $\mathrm{B}$, and $\mathrm{C}$ (from east to west). At higher angular resolution, however, their source A breaks down into two sub-condensation. They interpret the easternmost (and weakest) of these two subcondensations as the protostar VLA 1623 itself, and the western sub-condensation, as well as the sources B and C, as knots along a jet driven by VLA 1623. In more recent publications, the two sub-condensations within source A of Bontemps \& Andre (1997) have usually been referred to as VLA 1623A and VLA 1623B (e.g., Ward-Thompson et al. 2011; Murillo \& Lai 2013), while source B of Bontemps \& Andre (1997) has been renamed VLA $1623 \mathrm{~W}$; source $\mathrm{C}$ has, to our knowledge, never been detected again. While there is general agreement that VLA $1623 \mathrm{~A}$ is a protostar, the nature of VLA 1623B and VLA $1623 \mathrm{~W}$ is still debated. ${ }^{15}$ Looney et al. (2000) obtained dust observations at $\lambda=2.7 \mathrm{~mm}$ and concluded that VLA 1623B was, most likely, a stellar companion of VLA 1623A. A similar

15 Note, however, that both have measurable proper motions similar to those of other sources in Ophiuchus (J. L. Rivera et al., in preparation). Thus, they are associated with that region: neither is an related background or foreground objects. conclusion was reached by Murillo \& Lai (2013) based on SubMillimeter Array observations. The latter authors detected VLA $1623 \mathrm{~W}$ as a bright point source in the MIPS $24 \mu \mathrm{m}$ data. This strongly suggests that it might also be a protostellar source, making VLA 1623 a triple system. However, some properties of the radio emission from VLA 1623B are more easily explained if it is interpreted as a knot along the jet (Ward-Thompson et al. 2011).

VLA $1623 \mathrm{~A}, \mathrm{~B}$, and $\mathrm{W}$ are detected in our observations as GBS-VLA J162626.39-242430.9, GBS-VLA J162626.31242430.3, and GBS-VLA J162625.62-242429.2, respectively. Source C of Bontemps \& Andre (1997), however, is not seen in our data. Both VLA 1623A and VLA 1623B have positive spectral indices of order 0.8 , more typical of protostellar sources than of outflow features. VLA $1623 \mathrm{~W}$, on the other hand, has a spectral index $(-0.2 \pm 0.6)$ consistent with the optically thin free-free emission expected from an outflow knot (e.g., Pech et al. 2010). Interestingly, however, the separations between VLA 1623A and VLA 1623B (1".2) and between VLA 1623A and VLA $1623 \mathrm{~W}\left(10^{\prime \prime} .5\right)$ have not changed appreciably during the $\sim 15$ yr separating the observations reported by Bontemps \& Andre (1997) and those described here. This sets an upper limit of about $4 \mathrm{~km} \mathrm{~s}^{-1}$ on their relative motion. While this would be consistent with the expected orbital motion in a solar mass multiple system, it is harder to reconcile with sources B and W being outflow knots.

\subsection{Radio Detections at the Stellar-brown Dwarf Boundary}

We detect radio emission from two brown dwarf candidates: GBS-VLA J162722.96-242236.6 is associated with a brown 
dwarf reported and documented by Marsh et al. (2010), while GBS-VLA J162715.70-243845.6 is a brown dwarf candidate (Alves de Oliveira et al. 2010) located in the southernmost component in the triple system WL 20. We note that the association of the radio source with the brown dwarf candidate in WL 20 (rather than with the higher-mass members of the system) is secure since the angular offset between the position of the radio source and the nominal position of the brown dwarf candidate is 0.14 , while its separations from the other stars in the system are 2".42 and 3".31. We also detected radio emission from two $\mathrm{M}$ stars near the brown dwarf boundary: GBS-VLA J162556.09-243015.3 is an M5 YSO of Class III, while GBSVLA J162759.95-244819.5 is an M4.75 weak line T Tauri star.

We will discuss these cases in more details in a forthcoming dedicated publication, but would like to note here that in three of these four objects near the brown dwarf boundary, the radio emission shows clear indications (high levels of variability and/or a negative spectral index) of being non-thermal. Only in the case of GBS-VLA J162556.09-243015.3 is the emission more likely thermal (free-free) since the spectral index is around zero and the radio flux quite steady. Our detections show that YSOs near or beyond the brown dwarf boundary can be detectable radio emitters even at a distance of more than $100 \mathrm{pc}$. Like their older counterparts (e.g., McLean et al. 2011; Ravi et al. 2011), they appear to have a large radio to bolometric luminosity ratio.

\subsection{Non-thermal Radio Emission from Class I Objects}

Protostellar objects (of Class 0 and I) are expected to have strong winds producing thermal bremsstrahlung (free-free) emission that is optically thick at least in the dense region immediately surrounding the protostar (e.g., Rodríguez 1999). In this situation, even if the protostar itself emitted non-thermal (gyrosynchrotron) radiation, it ought to be absorbed by the ionized wind and should not reach the observer. It is noteworthy, however, that a small number of Class I sources have been found to be non-thermal emitters. Perhaps the most robust cases are IRS5 in Corona Australis (Feigelson et al. 1998; Deller et al. 2013), EC 95 in Serpens (Dzib et al. 2010), and (more marginally) YLW 15 in Ophiuchus (Forbrich et al. 2007). The detectability of non-thermal emission in these objects might be due to a favorable geometry (e.g., when the protostar is seen nearly pole-on or nearly edge-on, the free-free opacity might be reduced; Forbrich et al. 2007), or to tidal clearing of circumstellar material in a tight binary system (Dzib et al. 2010).

We find two possible non-thermal Class I sources in our sample. On the one hand, GBS-VLA J162726.90-244050.8 corresponds to YLW 15 which, as we just mentioned, is one of the known candidates non-thermal Class I sources. In our observations, its spectral index is negative (suggesting a nonthermal process) but its flux is almost constant. Forbrich et al. (2007) marginally detected one of the components in YLW 15 during VLBI observations at $8.4 \mathrm{GHz}$, at a level of $145 \mu \mathrm{Jy}$. An independent, more robust, VLBI detection would be necessary to confirm the non-thermal nature of the source. The other target of interest in this context is GBS-VLA J163200.97-245643.3 which is associated with the Class I source WLY 2-67. While it has a positive spectral index, it exhibits significant variability and it is found to be significantly circularly polarized $(11 \%$ and $25 \%$ at 4.5 and $7.5 \mathrm{GHz}$, respectively). This provides a clear indication that the radio emission is non-thermal. A VLBI detection should be attempted of this target, as it would provide

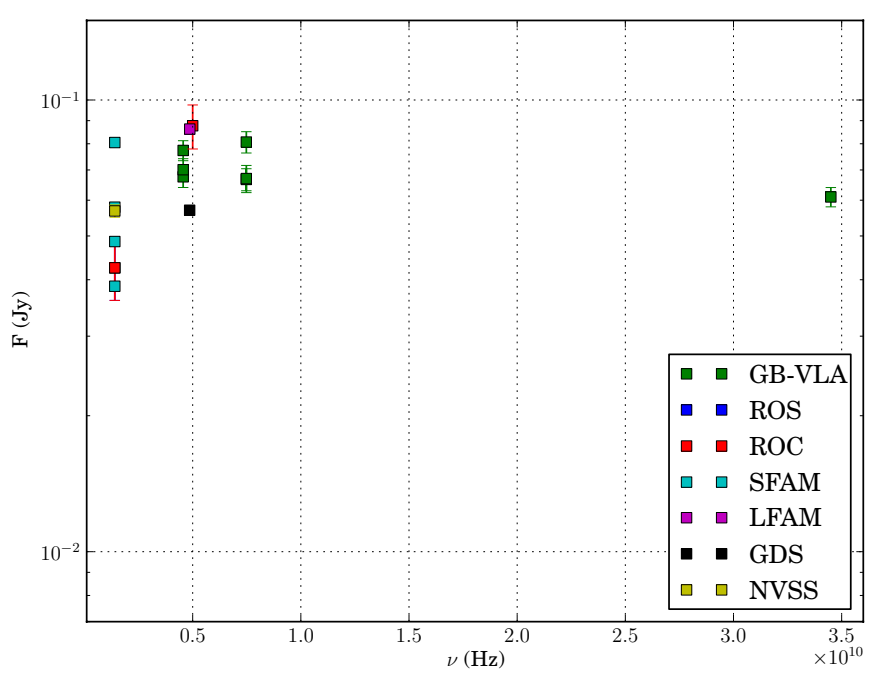

Figure 8. Spectral energy distribution of the extragalactic source GBS-VLA J162700.00-242640.3. The points correspond to the catalogs given in the legend (GBS-VLA correspond to the present observations, whereas the other symbols are the same as given in footnote "d" of Table 3 .

(A color version of this figure is available in the online journal.)

a direct and independent confirmation of the non-thermal origin of the emission.

\subsection{GBS-VLA J162700.00-242640.3: A New Calibrator for Ophiuchus}

One of the known extragalactic targets detected here (GBSVLA J162700.00-242640.3) is found to have a flux of order 71 mJy both at 4.5 and $7.5 \mathrm{GHz}$, to exhibit very little variability $(\sim 15 \%$; Table 1$)$ and to be unresolved in our VLA data. It has been detected in a number of previous radio observations (Table 3) as well as in high-resolution $K a$-band observations (at $v=32.5 \mathrm{GHz}$ ) that we obtained in 2011 in the BnA configuration of the VLA. The spectral energy distribution constructed from all available radio data (Figure 8) shows that it is a flat spectrum source. We have observed it with the Very Long Baseline Array (VLBA) at $v=8.4 \mathrm{GHz}$, and detected it as an unresolved $70 \mathrm{mJy}$ source. Since GBS-VLA J162700.00-242640.3 is located in the direction of the Ophiuchus core (Figure 2), it ought to be used as main gain calibrator for any future interferometric (conventional or long baseline) observations of the Ophiuchus complex.

\section{CONCLUSIONS AND PERSPECTIVES}

In this paper, we have reported on radio observations of the Ophiuchus complex that largely surpass all such previous observations thanks to a combination of high sensitivity, good angular resolution, and large field of view. A total of 189 sources were detected, 56 of them associated with known young stellar sources, and 4 with known extragalactic objects; the other 129 remain unclassified, but most of them are certainly extragalactic background sources. Most of the young stars detected at radio wavelengths have spectral types of $\mathrm{K}$ or $\mathrm{M}$, but we also detect two brown dwarf candidates.

Interestingly, at least half of these young stars are non-thermal (gyrosynchrotron) sources, with active magnetized coronas. These sources are excellent targets for future astrometric observations with VLBI instruments (similar to but much more extensive than those reported by Loinard et al. 2008) that would enable an accurate determination of the distance, kinematics, 
and internal structure of the Ophiuchus region. Such observations would be greatly aided by the detection, reported here, of an adequate gain calibrator (GBS-VLA J162700.00-242640.3) located toward the Ophiuchus core.

L.L. is grateful to the von Humboldt Stiftung for financial support. S.D., L.L., L.F.R., G.N.O., G.P., and J.L.R. acknowledge the financial support of DGAPA, UNAM, and CONACyT, México. The National Radio Astronomy Observatory is operated by Associated Universities, Inc., under cooperative agreement with the National Science Foundation. CASA is developed by an international consortium of scientists based at the National Radio Astronomical Observatory (NRAO), the European Southern Observatory (ESO), the National Astronomical Observatory of Japan (NAOJ), the CSIRO Australia Telescope National Facility (CSIRO/ATNF), and the Netherlands Institute for Radio Astronomy (ASTRON) under the guidance of NRAO. This research has made use of the SIMBAD database, operated at CDS, Strasbourg, France.

\section{REFERENCES}

Alves de Oliveira, C., Moraux, E., Bouvier, J., et al. 2010, A\&A, 515, A75 Andre, P., Deeney, B. D., Phillips, R. B., \& Lestrade, J.-F. 1992, ApJ, 401, 667 André, P., Montmerle, T., \& Feigelson, E. D. 1987, AJ, 93, 1182

Andre, P., Ward-Thompson, D., \& Barsony, M. 1993, ApJ, 406, 122

Andrews, S. M., \& Williams, J. P. 2007, ApJ, 671, 1800

Barsony, M., Haisch, K. E., Marsh, K. A., \& McCarthy, C. 2012, ApJ, 751, 22 Benz, A. O., \& Güdel, M. 1994, A\&A, 285, 621

Berger, E., Basri, G., Fleming, T. A., et al. 2010, ApJ, 709, 332

Bontemps, S., \& Andre, P. 1997, in IAU Symp. 182, Herbig-Haro Objects and the Birth of Low Mass Stars, ed. F. Malbet \& A. Castets (Cambridge: Cambridge Univ. Press), 63

Bouvier, J., \& Appenzeller, I. 1992, A\&AS, 92, 481

Briceño, C., Hartmann, L., Stauffer, J., \& Martín, E. 1998, AJ, 115, 2074

Briceño, C., Hartmann, L. W., Stauffer, J. R., et al. 1997, AJ, 113, 740

Brown, R. L., \& Zuckerman, B. 1975, ApJL, 202, L125

Chandler, C. J., Brogan, C. L., Shirley, Y. L., \& Loinard, L. 2005, ApJ, 632, 371

Cieza, L., Padgett, D. L., Stapelfeldt, K. R., et al. 2007, ApJ, 667, 308

Condon, J. J., Cotton, W. D., Greisen, E. W., et al. 1998, AJ, 115, 1693

Cutri, R. M., Skrutskie, M. F., van Dyk, S., et al. 2003, The IRSA 2MASS All-Sky Point Source Catalog, NASA/IPAC Infrared Science Archive, http://irsa.ipac.caltech.edu/applications/Gator/

Cutri, R. M., Wright, E. L., Conrow, T., et al. 2012, Explanatory Supplement to the WISE All-Sky Data Release Products, 1

Deller, A. T., Forbrich, J., \& Loinard, L. 2013, A\&A, 552, A51

Downes, J. J., Briceño, C., Hernández, J., et al. 2008, AJ, 136, 51

Dulk, G. A. 1985, ARA\&A, 23, 169

Dzib, S., Loinard, L., Mioduszewski, A. J., et al. 2010, ApJ, 718, 610

Erickson, K. L., Wilking, B. A., Meyer, M. R., Robinson, J. G., \& Stephenson, L. N. 2011, AJ, 142, 140

Evans, N. J., II, Dunham, M. M., Jørgensen, J. K., et al. 2009, ApJS, 181, 321

Falgarone, E., \& Gilmore, W. 1981, A\&A, 95, 32

Feigelson, E. D., Carkner, L., \& Wilking, B. A. 1998, ApJL, 494, L215

Fomalont, E. B., Windhorst, R. A., Kristian, J. A., \& Kellerman, K. I. 1991, AJ, 102,1258

Forbrich, J., Massi, M., Ros, E., Brunthaler, A., \& Menten, K. M. 2007, A\&A, 469, 985

Forbrich, J., Posselt, B., Covey, K. R., \& Lada, C. J. 2010, ApJ, 719, 691

Gagné, M., Skinner, S. L., \& Daniel, K. J. 2004, ApJ, 613, 393

Geers, V. C., Augereau, J.-C., Pontoppidan, K. M., et al. 2006, A\&A, 459, 545
Girart, J. M., Curiel, S., Rodríguez, L. F., et al. 2004, AJ, 127, 2969

Girart, J. M., Rodríguez, L. F., \& Curiel, S. 2000, ApJL, 544, L153

Grosso, N., Montmerle, T., Bontemps, S., André, P., \& Feigelson, E. D. 2000 , A\&A, 359, 113

Güdel, M., \& Benz, A. O. 1993, ApJL, 405, L63

Hovatta, T., Lehto, H. J., \& Tornikoski, M. 2008, A\&A, 488, 897

Hsu, W.-H., Hartmann, L., Allen, L., et al. 2013, ApJ, 764, 114

Imanishi, K., Koyama, K., \& Tsuboi, Y. 2001, ApJ, 557, 747

Imanishi, K., Nakajima, H., Tsujimoto, M., Koyama, K., \& Tsuboi, Y. 2003, PASJ, 55, 653

Johnstone, D., Wilson, C. D., Moriarty-Schieven, G., et al. 2000, ApJ, 545,327

Kessler-Silacci, J., Augereau, J.-C., Dullemond, C. P., et al. 2006, ApJ, 639, 275

Kroupa, P. 2001, MNRAS, 322, 231

Lahuis, F., van Dishoeck, E. F., Blake, G. A., et al. 2007, ApJ, 665, 492

Leous, J. A., Feigelson, E. D., Andre, P., \& Montmerle, T. 1991, ApJ, 379, 683

Lestrade, J.-F., Phillips, R. B., Preston, R. A., \& Gabuzda, D. C. 1991, BAAS, 23,880

Lodieu, N., Dobbie, P. D., \& Hambly, N. C. 2011, A\&A, 527, A24

Loinard, L., Chandler, C. J., Rodríguez, L. F., et al. 2007, ApJ, 670, 1353

Loinard, L., Torres, R. M., Mioduszewski, A. J., \& Rodríguez, L. F. 2008, ApJL, 675, L29

Loinard, L., Zapata, L. A., Rodríguez, L. F., et al. 2013, MNRAS, 430, L10

Looney, L. W., Mundy, L. G., \& Welch, W. J. 2000, ApJ, 529, 477

Marsh, K. A., Plavchan, P., Kirkpatrick, J. D., et al. 2010, ApJ, 719, 550

Martin, E. L., Montmerle, T., Gregorio-Hetem, J., \& Casanova, S. 1998, MNRAS, 300, 733

Martin, E. L., Rebolo, R., \& Zapatero-Osorio, M. R. 1996, ApJ, 469, 706

McClure, M. K., Furlan, E., Manoj, P., et al. 2010, ApJS, 188, 75

McLean, M., Berger, E., Irwin, J., Forbrich, J., \& Reiners, A. 2011, ApJ, 741, 27

Montmerle, T., Koch-Miramond, L., Falgarone, E., \& Grindlay, J. E. 1983, ApJ, 269,182

Motte, F., Andre, P., \& Neri, R. 1998, A\&A, 336, 150

Murillo, N. M., \& Lai, S.-P. 2013, ApJL, 764, L15

Ozawa, H., Grosso, N., \& Montmerle, T. 2005, A\&A, 429, 963

Padgett, D. L., Rebull, L. M., Stapelfeldt, K. R., et al. 2008, ApJ, 672, 1013

Panagia, N. 1973, AJ, 78, 929

Pech, G., Loinard, L., Chandler, C. J., et al. 2010, ApJ, 712, 1403

Phillips, R. B., Lonsdale, C. J., \& Feigelson, E. D. 1991, ApJ, 382, 261

Pillitteri, I., Sciortino, S., Flaccomio, E., et al. 2010, A\&A, 519, A34

Poppel, W. 1997, FCPh, 18, 1

Ravi, V., Hallinan, G., Hobbs, G., \& Champion, D. J. 2011, ApJL, 735, L2

Ridge, N. A., Di Francesco, J., Kirk, H., et al. 2006, AJ, 131, 2921

Rodríguez, L. F. 1999, in Proc. Star Formation 1999, ed. T. Nakamoto (Nobeyama: Nobeyama Radio Observatory), 257

Schlieder, J. E., Lépine, S., Rice, E., et al. 2012, AJ, 143, 114

Sciortino, S., Pillitteri, I., Damiani, F., et al. 2006, in Proc. The X-ray Universe 2005, ed. A. Wilson (ESA SP-604; Noordwijk: ESA), 111

Skrutskie, M. F., Cutri, R. M., Stiening, R., et al. 2006, AJ, 131, 1163

Slesnick, C. L., Carpenter, J. M., \& Hillenbrand, L. A. 2006, AJ, 131, 3016

Stanke, T., Smith, M. D., Gredel, R., \& Khanzadyan, T. 2006, A\&A, 447, 609

Stine, P. C., Feigelson, E. D., Andre, P., \& Montmerle, T. 1988, AJ, 96, 1394

Torres, C. A. O., Quast, G. R., da Silva, L., et al. 2006, A\&A, 460, 695

Voges, W., Aschenbach, B., Boller, T., et al. 1999, A\&A, 349, 389

Wahhaj, Z., Cieza, L., Koerner, D. W., et al. 2010, ApJ, 724, 835

Ward-Thompson, D., Kirk, J. M., Greaves, J. S., \& André, P. 2011, MNRAS, 415,2812

Wilking, B. A., Bontemps, S., Schuler, R. E., Greene, T. P., \& André, P. 2001, ApJ, 551, 357

Wilking, B. A., Gagné, M., \& Allen, L. E. 2008, in Handbook of Star Forming Regions, Vol. II: The Southern Sky, ed. B. Reipurth (ASP Monograph Publ. Vol. 5; San Francisco, CA: ASP), 351

Wilking, B. A., Meyer, M. R., Robinson, J. G., \& Greene, T. P. 2005, AJ, 130,1733

Wootten, A. 1989, ApJ, 337, 858

Young, C. H., Bourke, T. L., Young, K. E., et al. 2006, AJ, 132, 1998 